\title{
ERROR ANALYSIS OF HIGH-ORDER SPLITTING METHODS FOR NONLINEAR EVOLUTIONARY SCHRÖDINGER EQUATIONS AND APPLICATION TO THE MCTDHF EQUATIONS IN ELECTRON DYNAMICS
}

\author{
Othmar $\mathrm{Koch}^{1}, \mathrm{Christof} \mathrm{Neuhauser}^{2}$ and Mechthild Thalhammer ${ }^{3}$
}

\begin{abstract}
In this work, the error behaviour of high-order exponential operator splitting methods for the time integration of nonlinear evolutionary Schrödinger equations is investigated. The theoretical analysis utilises the framework of abstract evolution equations on Banach spaces and the formal calculus of Lie derivatives. The general approach is substantiated on the basis of a convergence result for exponential operator splitting methods of (nonstiff) order $p$ applied to the multi-configuration timedependent Hartree-Fock (MCTDHF) equations, which are associated with a model reduction for highdimensional linear Schrödinger equations describing free electrons that interact by Coulomb force. Provided that the analytical solution of the MCTDHF equations constituting a system of coupled linear ordinary differential equations and low-dimensional nonlinear partial differential equations satisfies suitable regularity requirements, convergence of order $p-1$ in the $H^{1}$ Sobolev norm and convergence of order $p$ in the $L^{2}$ norm is proven. An analogous result follows for the cubic nonlinear Schrödinger equation, which is also illustrated by a numerical experiment.
\end{abstract}

Mathematics Subject Classification. 65L05, 65M12, 65J15.

Received August 10, 2011. Revised February 14, 2012.

Published online July 9, 2013.

\section{INTRODUCTION}

Numerous models in the physical sciences are described by nonlinear partial differential equations that involve operators of different nature and stiffness properties, which suggests separate treatment with respect to both, space and time discretisation. In order to accommodate for these situations, time-splitting methods have become

\footnotetext{
Keywords and phrases. Nonlinear evolution equations, time-dependent nonlinear Schrödinger equations, multi-configuration time-dependent Hartree-Fock (MCTDHF) equations, high-order exponential operator splitting methods, local error expansion, convergence.

1 Institute for Analysis and Scientific Computing, Vienna University of Technology, Wiedner Hauptstrasse 8-10, 1040 Wien, Austria. othmar@othmar-koch.org

2 Institut für Mathematik, Leopold-Franzens Universität Innsbruck, Technikerstrasse 13/VII, 6020 Innsbruck, Austria. christof.neuhauser@uibk.ac.at

3 Institut für Mathematik und Rechneranwendung, Universität der Bundeswehr München, Werner-Heisenberg-Weg 39, 85577

Neubiberg, Germany. mechthild.thalhammer@uibk.ac.at
} 
popular in the last decades, where the most classical examples are the first-order Lie-Trotter [51] and the second-order Strang [46] splitting method specified below, see also [18,35] for a detailed exposition on the construction and theoretical analysis of splitting methods for nonstiff problems.

A variety of works provide numerical simulations that illustrate the favourable behaviour of time-splitting methods for nonlinear Schrödinger equations; as a small selection, we mention the contributions $[3,4,13,14,44]$ on time-dependent Gross-Pitaevskii systems describing multi-component Bose-Einstein condensates and refer to further work by W. Bao and co-authors. Numerical comparisons given in [13] show the superior behaviour of higher-order splitting methods regarding efficiency, accuracy, and the preservation of physically relevant quantities when smaller tolerances are required or long-term computations are carried out: Standard time integration methods such as explicit Runge-Kutta methods are outperformed, mainly because of their poor stability properties for stiff problems; furthermore, the numerical experiments illustrate the favourable behaviour of optimised fourth- or sixth-order schemes compared to the second-order Strang splitting method.

However, so far it remains open to confirm numerical evidence by a rigorous analysis for discretisations based on high-order exponential operator splitting methods. A seminal theoretical work is [34] providing a rigorous stability and error analysis of the second-order Strang splitting method for the Schrödinger-Poisson and the cubic Schrödinger equation. In [17] the convergence analysis has been extended to full discretisation by the Strang splitting method and Hermite spectral collocation applied to the Gross-Pitaevskii equation; the derivation of the convergence result substantially relies on a global error bound for the time-discrete solution deduced in [34].

In the present work, our aim is to investigate the error behaviour of high-order exponential operator splitting methods for the efficient time integration of nonlinear evolutionary Schrödinger equations. In particular, we are concerned with the derivation of a convergence result under optimal regularity requirements on the analytical solution. The present work is in the lines of [34]: Main tools in our analysis are a suitable local error expansion extending the result for the linear case [48] and bounds for iterated Lie-commutators. To the best of our knowledge, this is for the first time that such an error analysis is given for high-order time-splitting methods in the context of nonlinear evolution equations. The general approach is substantiated on the basis of the multiconfiguration time-dependent Hartree-Fock (MCTDHF) equations in electron dynamics [27-29] and as special case also includes the cubic Schrödinger equation.

The manuscript is organised as follows. In Section 2, we deduce a local error expansion for high-order exponential operator splitting methods, which is a fundamental tool in the convergence analysis for a particular application. For this purpose, we introduce a general analytical framework of nonlinear evolutionary problems and employ the formal calculus of Lie derivatives. For the convenience of the reader who is not familar with the concept of Lie-derivatives, basic definitions and auxiliary results needed in the derivation of our local error expansion are collected in Appendix A. In Section 3, as an application, we analyse the error behaviour of high-order variational splitting methods for the approximate solution of time-dependent linear Schrödinger equations that serve as models for systems of unbound fermions interacting by Coulomb force; variational splitting methods are equivalent to exponential operator splitting methods for the time integration of the MCTDHF equations under a special gauge condition. In Sections 3.1 and 3.2, we introduce the MCTDHF equations and their time discretisation by exponential operator splitting methods. In Section 3.3, we state our main result on the convergence behaviour of high-order splitting methods for the MCTDHF equations and sketch its proof. Provided that the exact solution satisfies suitable regularity requirements, we prove convergence of order $p-1$ in the $H^{1}$ Sobolev norm and convergence of order $p$ in the $L^{2}$ norm for a splitting method of (nonstiff) order $p$. Our theoretical analysis generalises [24, 26-29,32,34,43,48] and utilises the framework of abstract evolution equations on Banach spaces, the formal calculus of Lie derivatives, and bounds for iterated Lie-commutators of nonlinear operators. The assumptions on the involved nonlinear operators associated with the MCTDHF equations are verified in Section 3.4. As a corollary, we obtain the corresponding result for the less involved cubic Schrödinger equation, which is also illustrated by a numerical example. Conclusions and an outlook to future work are finally given in Section 4. 


\subsection{Notation}

Throughout, we employ the following abbreviations and conventions. The cardinality of a (finite) set $M$, that is, the number of elements in $M$, is denoted by $|M|$. As common usage, a sum with upper limit smaller than the lower one is equal to zero; similarly, a product is equal to one whenever the upper limit is smaller than the lower one. We use the standard multi-index notation $\mu=\left(\mu_{1}, \ldots, \mu_{k}\right) \in \mathbb{N}^{k}$ and the vector notation $\xi=\left(\xi_{1}, \ldots, \xi_{k}\right) \in \mathbb{R}^{k}$; further, we write in short $|\mu|=\mu_{1}+\cdots+\mu_{k}$ as well as $\partial_{\xi}^{\mu}=\partial_{\xi_{1}}^{\mu_{1}} \ldots \partial_{\xi_{k}}^{\mu_{k}}$ and

$$
\int_{\Omega} f(\xi) \mathrm{d} \xi=\int_{\Omega} f\left(\xi_{1}, \ldots, \xi_{k}\right) \mathrm{d} \xi_{k} \ldots \mathrm{d} \xi_{1}
$$

for a domain $\Omega \subset \mathbb{R}^{k}$. The Lebesgue space $L^{2}(\Omega)$ of square integrable functions $f: \Omega \rightarrow \mathbb{C}$ is endowed with scalar product and associated norm given by

$$
(f \mid g)_{L^{2}(\Omega)}=\int_{\Omega} \overline{f(\xi)} g(\xi) \mathrm{d} \xi, \quad\|f\|_{L^{2}(\Omega)}=\sqrt{(f \mid f)_{L^{2}(\Omega)}}, \quad f, g \in L^{2}(\Omega),
$$

and, in particular, for a selfadjoint operator $\mathscr{L}: D(\mathscr{L}) \subset L^{2}(\Omega) \rightarrow L^{2}(\Omega)$, we let

$$
(f|\mathscr{L}| g)_{L^{2}(\Omega)}=(\mathscr{L} f \mid g)_{L^{2}(\Omega)}=(f \mid \mathscr{L} g)_{L^{2}(\Omega)}, \quad f, g \in D(\mathscr{L})
$$

the Banach space $L^{\infty}(\Omega)$ is endowed with the norm $\|f\|_{L^{\infty}(\Omega)}=\operatorname{ess} \sup \{|f(x)|: x \in \Omega\}$. The Sobolev space $H^{m}(\Omega)$ comprises all functions with partial derivatives up to order $m \geq 0$ contained in $L^{2}(\Omega)$; the associated norm is denoted by $\|\cdot\|_{H^{m}(\Omega)}$, and, in particular, it holds $H^{0}(\Omega)=L^{2}(\Omega)$. Detailed information on Sobolev spaces is found in [2]. Besides, we denote by $I$ the identity operator and by $C$ a generic constant; for a normed space $\left(X,\|\cdot\|_{X}\right)$, the associated operator norm is denoted by $\|\cdot\|_{X \leftarrow X}$.

\section{A LOCAL ERROR EXPANSION}

In this section, we deduce a local error expansion of high-order exponential operator splitting methods applied to a nonlinear evolutionary problem of the form

$$
u^{\prime}(t)=A(u(t))+B(u(t)), \quad 0 \leq t \leq T, \quad u(0) \text { given, }
$$

involving unbounded nonlinear operators $A: D(A) \subset X \rightarrow X$ and $B: D(B) \subset X \rightarrow X$; throughout, we tacitly assume that the domains $D(A)$ and $D(B)$ are suitably chosen subsets of the underlying Banach space $\left(X,\|\cdot\|_{X}\right)$ with non-empty intersection $D(A) \cap D(B) \neq \emptyset$.

Employing the formal calculus of Lie derivatives introduced in Appendix A, any high-order exponential operator splitting method for $(2.1)$ can be written in the following form. Let $0=t_{0}<t_{1}<\cdots<t_{N} \leq T$ denote the time grid points with associated stepsizes $h_{n}=t_{n+1}-t_{n}$ for $0 \leq n \leq N-1$ and $h=\max \left\{h_{n}: 0 \leq n \leq N-1\right\}$ the maximal time stepsize. Starting from an initial value $u_{0} \approx u(0)$, numerical approximations to the exact solution values are determined from the recurrence relation

$$
u_{n+1}=\mathscr{S}\left(h_{n}, u_{n}\right) \quad \approx u\left(t_{n+1}\right)=\mathscr{E}\left(h_{n}, u\left(t_{n}\right)\right), \quad 0 \leq n \leq N-1 .
$$

Provided that the (real) method coefficients $\left(a_{j}, b_{j}\right)_{j=1}^{s}$ satisfy certain order conditions, see [48] and references given therein, the nonlinear splitting operator is an approximation of order $p \geq 1$ to the exact solution operator

$$
\mathscr{S}(t, \cdot)=\prod_{j=1}^{s} \mathrm{e}^{a_{s+1-j} t D_{A}} \mathrm{e}^{b_{s+1-j} t D_{B}} \quad \approx \mathscr{E}(t, \cdot)=\mathrm{e}^{t D_{A+B}}, \quad 0 \leq t \leq T ;
$$

here, the product is defined downwards. 
In particular, for the choice

$$
s=2, \quad a_{1}=0, \quad a_{2}=1, \quad b_{1}=\frac{1}{2}=b_{2},
$$

we retain the second-order Strang type splitting method studied in [32] for the Schrödinger-Poisson equation and the cubic Schrödinger equation, in [17] for the Gross-Pitaevskii equation, and in $[24,25,29]$ in the context of the MCTDHF equations.

In order to deduce a suitable representation of the defect operator

$$
\mathscr{D}(t, v)=\mathscr{S}(t, v)-\mathscr{E}(t, v), \quad 0 \leq t \leq T,
$$

we first expand the exact evolution operator and the splitting operator by the nonlinear variation-of-constants formula (A.4) and the recurrence relation (A.3i); both expansions are related through quadrature formulas for multiple integrals. A further expansion of the integrand then yields the desired local error representation involving iterated Lie-commutators of nonlinear operators; from this representation the nonstiff order conditions are retained.

We point out that by the formal calculus of Lie-derivatives the expansion for the nonlinear case carries over from the linear case [48] by exchanging the (linear) operator $F$ with the Lie derivative $D_{F}$ and reversing the order in all terms which involve the composition of operators. For the reader's convenience, we illustrate the employed mechanisms by non-complex cases, see also Appendix A.

Step 1. Expansion of the evolution operator. As a first step, we use the nonlinear variation-of-constants formula (A.4)

$$
\mathscr{E}(t, v)=\mathrm{e}^{t D_{A+B}} v=\mathrm{e}^{t D_{A}} v+\int_{0}^{t} \mathrm{e}^{\tau_{1} D_{A+B}} D_{B} \mathrm{e}^{\left(t-\tau_{1}\right) D_{A}} v \mathrm{~d} \tau_{1}, \quad 0 \leq t \leq T
$$

considerations as in the proof of Theorem A.1 further imply

$$
\mathrm{e}^{\tau_{1} D_{A+B}} D_{B} \mathrm{e}^{\left(t-\tau_{1}\right) D_{A}} v-\mathrm{e}^{\tau_{1} D_{A}} D_{B} \mathrm{e}^{\left(t-\tau_{1}\right) D_{A}} v=\int_{0}^{\tau_{1}} \mathrm{e}^{\tau_{2} D_{A+B}} D_{B} \mathrm{e}^{\left(\tau_{1}-\tau_{2}\right) D_{A}} D_{B} \mathrm{e}^{\left(t-\tau_{1}\right) D_{A}} v \mathrm{~d} \tau_{2},
$$

see (A.5), and hence the representation

$$
\mathscr{E}(t, v)=\mathrm{e}^{t D_{A}} v+\int_{0}^{t} \mathrm{e}^{\tau_{1} D_{A}} D_{B} \mathrm{e}^{\left(t-\tau_{1}\right) D_{A}} v \mathrm{~d} \tau_{1}+\int_{0}^{t} \int_{0}^{\tau_{1}} \mathrm{e}^{\tau_{2} D_{A+B}} D_{B} \mathrm{e}^{\left(\tau_{1}-\tau_{2}\right) D_{A}} D_{B} \mathrm{e}^{\left(t-\tau_{1}\right) D_{A}} v \mathrm{~d} \tau_{2} \mathrm{~d} \tau_{1}
$$

is obtained. Proceeding by induction finally yields the following expansion for the exact evolution operator associated with (2.1)

$$
\begin{gathered}
\mathscr{E}(t, v)=\mathrm{e}^{t D_{A}} v+\sum_{k=1}^{p} \mathscr{I}_{k}(t, v)+\mathscr{R}_{p+1}^{(1)}(t, v), \quad 0 \leq t \leq T, \\
\mathscr{I}_{k}(t, v)=\int_{T_{k}} g_{k}(\tau, v) \mathrm{d} \tau, \quad \mathscr{R}_{p+1}^{(1)}(t, v)=\int_{T_{p+1}} \mathrm{e}^{\tau_{p+1} D_{A+B}} f_{p+1}(\tau, v) \mathrm{d} \tau, \\
T_{k}=\left\{\tau=\left(\tau_{1}, \tau_{2}, \ldots, \tau_{k}\right) \in \mathbb{R}^{k}: 0 \leq \tau_{k} \leq \ldots \leq \tau_{1} \leq \tau_{0}=t\right\}, \\
f_{k}(\tau, v)=\prod_{\ell=1}^{k}\left(D_{B} \mathrm{e}^{\left(\tau_{\ell-1}-\tau_{\ell}\right) D_{A}}\right) v, \quad g_{k}(\tau, v)=\mathrm{e}^{\tau_{k} D_{A}} f_{k}(\tau, v), \quad \tau \in T_{k} .
\end{gathered}
$$


TABLE 1. Coefficients $\alpha_{\lambda_{\mu}}=\alpha_{\lambda_{\mu_{1}} \lambda_{\mu_{2}} \ldots \lambda_{\mu_{k}}}$ for $\mu=\left(\mu_{1}, \mu_{2}, \ldots, \mu_{k}\right)$.

\begin{tabular}{|c|c|c|}
\hline$k$ & $\mu$ & $\alpha_{\lambda_{\mu}}$ \\
\hline 1 & 1 & 1 \\
\hline \multirow[t]{2}{*}{2} & 11 & $1 / 2$ \\
\hline & 12 & 1 \\
\hline \multirow[t]{3}{*}{3} & 111 & $1 / 6$ \\
\hline & 112,122 & $1 / 2$ \\
\hline & 123 & 1 \\
\hline \multirow[t]{5}{*}{4} & 1111 & $1 / 24$ \\
\hline & 1112,1222 & $1 / 6$ \\
\hline & 1122 & $1 / 4$ \\
\hline & $1123,1223,1233$ & $1 / 2$ \\
\hline & 1234 & 1 \\
\hline \multirow[t]{7}{*}{5} & 11111 & $1 / 120$ \\
\hline & 11112,12222 & $1 / 24$ \\
\hline & 11122,11222 & $1 / 12$ \\
\hline & $11123,12223,12333$ & $1 / 6$ \\
\hline & $11223,11233,12233$ & $1 / 4$ \\
\hline & $11234,12234,12334,12344$ & $1 / 2$ \\
\hline & 12345 & 1 \\
\hline \multirow[t]{11}{*}{6} & 111111 & $1 / 720$ \\
\hline & 111112,122222 & $1 / 120$ \\
\hline & 111122,112222 & $1 / 48$ \\
\hline & 111222 & $1 / 36$ \\
\hline & $111123,122223,123333$ & $1 / 24$ \\
\hline & $111223,111233,112223,112333,122233,122333$ & $1 / 12$ \\
\hline & 112233 & $1 / 8$ \\
\hline & $111234,122234,123334,123444$ & $1 / 6$ \\
\hline & $112234,112334,112344,122334,122344,123344$ & $1 / 4$ \\
\hline & $112345,122345,123345,123445,123455$ & $1 / 2$ \\
\hline & 123456 & 1 \\
\hline
\end{tabular}

Step 2. Expansion of the splitting operator. On the other hand, a stepwise expansion of the splitting operator $(2.2 \mathrm{~b})$ by means of the recurrence relation (A.3i) yields the discrete analogue of $(2.4 \mathrm{a})$

$$
\begin{gathered}
\mathscr{S}(t, v)=\mathrm{e}^{t D_{A}} v+\sum_{k=1}^{p} \mathscr{Q}_{k}(t, v)+\mathscr{R}_{p+1}^{(2)}(t, v), \quad 0 \leq t \leq T, \\
\mathscr{Q}_{k}(t, v)=t^{k} \sum_{\lambda \in L_{k}} \alpha_{\lambda} \prod_{\ell=1}^{k} b_{\lambda_{\ell}} g_{k}\left(c_{\lambda} t, v\right), \\
c_{\lambda} t=\left(c_{\lambda_{1}} t, \ldots, c_{\lambda_{k}} t\right), \quad c_{j}=\sum_{\ell=1}^{j} a_{\ell}, \\
L_{k}=\left\{\lambda=\left(\lambda_{1}, \lambda_{2}, \ldots, \lambda_{k}\right) \in \mathbb{N}^{k}: 1 \leq \lambda_{k} \leq \ldots \leq \lambda_{1} \leq \lambda_{0}=s\right\} .
\end{gathered}
$$

Here, $\alpha_{\lambda}=\frac{1}{\nu_{1} ! \nu_{2} ! \ldots \nu_{l} !}$ holds for any $\lambda \in L_{k}$, written in the form

$$
\begin{gathered}
\lambda=\left(\lambda_{\kappa_{1}}, \lambda_{\kappa_{1}+1}, \ldots, \lambda_{\kappa_{1}+\left(\nu_{1}-1\right)}, \lambda_{\kappa_{2}}, \lambda_{\kappa_{2}+1}, \ldots, \lambda_{\kappa_{2}+\left(\nu_{2}-1\right)}, \ldots, \lambda_{\kappa_{l}}, \lambda_{\kappa_{l}+1}, \ldots, \lambda_{\kappa_{l}+\left(\nu_{l}-1\right)}\right), \\
\lambda_{\kappa_{1}}=\lambda_{\kappa_{1}+1}=\ldots=\lambda_{\kappa_{1}+\left(\nu_{1}-1\right)}>\lambda_{\kappa_{2}}=\lambda_{\kappa_{2}+1}=\ldots=\lambda_{\kappa_{2}+\left(\nu_{2}-1\right)}>\lambda_{\kappa_{l}}=\lambda_{\kappa_{l}+1}=\ldots=\lambda_{\kappa_{l}+\left(\nu_{l}-1\right)} .
\end{gathered}
$$

As an illustration, for $1 \leq k \leq 6$, the coefficients $\alpha_{\lambda}, \lambda \in L_{k}$, are stated in Table 1 . We also refer to [48], where the procedural method for the linear case is specified. 
Step 3. Derivatives of the integrand. We next determine higher-order partial derivatives of the integrand in $\mathscr{I}_{k}$, see (2.4a); a brief calculation yields

$$
\partial_{\tau}^{\mu} g_{k}(\tau, v)=\mathrm{e}^{\tau_{k} D_{A}} \prod_{\ell=1}^{k}\left(\operatorname{ad}_{D_{A}}^{\mu_{\ell}}\left(D_{B}\right) \mathrm{e}^{\left(\tau_{\ell-1}-\tau_{\ell}\right) D_{A}}\right) v, \quad \tau \in T_{k}, \quad \mu \in \mathbb{N}^{k} .
$$

Step 4. Taylor series expansion of the integrand. By comparison of (2.4a) and (2.4b), it is seen that the multiple sum $\mathscr{Q}_{k}$ is a quadrature formula approximation of the integral $\mathscr{I}_{k}$. Thus, employing a Taylor series of $g_{k}$ about zero and making use of (2.4c), the local error expansion summarised below in Theorem 2.1 results.

Theorem 2.1 (Local error expansion). We let $T_{k}=\left\{\tau=\left(\tau_{1}, \ldots, \tau_{k}\right) \in \mathbb{R}^{k}: 0 \leq \tau_{k} \leq \ldots \leq \tau_{1} \leq \tau_{0}=t\right\}$ and $L_{k}=\left\{\lambda=\left(\lambda_{1}, \ldots, \lambda_{k}\right) \in \mathbb{N}^{k}: 1 \leq \lambda_{k} \leq \ldots \leq \lambda_{1} \leq \lambda_{0}=s\right\}$. Provided that the condition $c_{s}=1$ is satisfied, where $c_{k}=a_{1}+a_{2}+\cdots+a_{k}$ for $1 \leq k \leq s$, the defect operator of the exponential operator splitting method (2.2) applied to the nonlinear evolutionary problem (2.1) admits the expansion

$$
\begin{gathered}
\mathscr{D}(t, v)=\sum_{k=1}^{p} \sum_{\substack{\mu \in \mathbb{N}^{k} \\
|\mu| \leq p-k}} \frac{1}{\mu !} t^{k+|\mu|} C_{k \mu} \prod_{\ell=1}^{k} \operatorname{ad}_{D_{A}}^{\mu_{\ell}}\left(D_{B}\right) \mathrm{e}^{t D_{A}} v+\mathscr{R}_{p+1}(t, v), \quad 0 \leq t \leq T, \\
C_{k \mu}=\sum_{\lambda \in L_{k}} \alpha_{\lambda} \prod_{\ell=1}^{k} b_{\lambda_{\ell}} c_{\lambda_{\ell}}^{\mu_{\ell}}-\prod_{\ell=1}^{k} \frac{1}{\mu_{\ell}+\cdots+\mu_{k}+k-\ell+1} .
\end{gathered}
$$

The remainder $\mathscr{R}_{p+1}(t, v)=\mathscr{R}_{p+1}^{(1)}(t, v)-\mathscr{R}_{p+1}^{(2)}(t, v)-\mathscr{R}_{p+1}^{(3)}(t, v)$ comprises the terms

$$
\begin{aligned}
& \mathscr{R}_{p+1}^{(1)}(t, v)=\int_{T_{p+1}} \mathrm{e}^{\tau_{p+1} D_{A+B}} \prod_{j=1}^{p+1}\left(D_{B} \mathrm{e}^{\left(\tau_{j-1}-\tau_{j}\right) D_{A}}\right) v \mathrm{~d} \tau, \\
& \mathscr{R}_{p+1}^{(2)}(t, v)=t^{p+1} \sum_{j=1}^{p+1} \sum_{\lambda \in L_{p+1}} \widetilde{\alpha}_{j \lambda} \prod_{\ell=1}^{\lambda_{p+1}-1}\left(\mathrm{e}^{a_{\lambda_{p+1}-\ell} t D_{A}} \mathrm{e}^{b_{\lambda_{p+1}-\ell} t D_{B}}\right) \\
& \times \mathrm{e}^{a_{\lambda_{p+1}} t D_{A}} \varphi_{j}\left(b_{\lambda_{p+1}} t D_{B}\right) \prod_{\ell=1}^{p+1}\left(b_{\lambda_{\ell}} D_{B} \mathrm{e}^{\left(c_{\lambda_{\ell-1}}-c_{\lambda_{\ell}}\right) t D_{A}}\right) v \\
& \mathscr{R}_{p+1}^{(3)}(t, v)=\sum_{k=1}^{p}\left(\int_{T_{k}} r_{k, p-k+1}(\tau, v) \mathrm{d} \tau-t^{k} \sum_{\lambda \in L_{k}} \alpha_{\lambda} \prod_{\ell=1}^{k} b_{\lambda_{\ell}} r_{k, p-k+1}\left(c_{\lambda} t, v\right)\right), \\
& r_{k, p-k+1}(\tau, v)=(p-k+1) \int_{0}^{1}(1-\zeta)^{p-k} \sum_{\substack{\mu \in \mathbb{N}^{k} \\
|\mu|=p-k+1}} \frac{(-1)^{|\mu|}}{\mu !} \tau^{\mu} \mathrm{e}^{\tau_{k} \zeta D_{A}} \times \prod_{\ell=1}^{k}\left(\operatorname{ad}_{D_{A}}^{\mu_{\ell}}\left(D_{B}\right) \mathrm{e}^{\left(\tau_{\ell-1}-\tau_{\ell}\right) \zeta D_{A}}\right) v \mathrm{~d} \zeta .
\end{aligned}
$$

The coefficients $\alpha_{\lambda}$ and $\widetilde{\alpha}_{\lambda}$ are computable by recurrencet.

\footnotetext{
${ }^{4} \mathrm{~A}$ MATLAB code to determine $\alpha_{\lambda}$ and $\widetilde{\alpha}_{\lambda}$ is available at http://techmath.uibk.ac.at/ mecht/research/research.html
} 


\section{Application to the MCTDHF equations}

In the following, we focus on the approximate solution of time-dependent linear Schrödinger equations which serve as models for systems of unbound fermions interacting by Coulomb force [30,40]

$$
\begin{aligned}
\mathrm{i} \partial_{t} \Psi(x, t)= & \mathscr{H}(x) \Psi(x, t), \quad x \in \mathbb{R}^{3 d}, \quad 0 \leq t \leq T, \\
& \mathscr{H}=\mathscr{A}+\mathscr{V}, \quad \mathscr{A}=-\frac{1}{2} \Delta .
\end{aligned}
$$

Here, the wave function $\Psi: \mathbb{R}^{3 d} \times[0, T] \rightarrow \mathbb{C}:(x, t)=\left(x_{1}, \ldots, x_{d}, t\right) \rightarrow \Psi(x, t)$ depends on the threedimensional spatial coordinates of $d$ particles and further on time; consequently, the linear differential operator $\mathscr{A}=-\frac{1}{2}\left(\Delta_{x_{1}}+\ldots+\Delta_{x_{d}}\right)$ comprises the Laplacian $\Delta_{x_{j}}=\partial_{x_{j 1}}^{2}+\partial_{x_{j 2}}^{2}+\partial_{x_{j 3}}^{2}$ with respect to $x_{j}=\left(x_{j 1}, x_{j 2}, x_{j 3}\right) \in$ $\mathbb{R}^{3}$ for $1 \leq j \leq d$. Taking into account the pairwise Coulomb interaction of the particles only, the multiplication operator $\mathscr{V}$ reduces to

$$
\mathscr{V}(x)=\sum_{1 \leq j<k \leq d} \frac{1}{\left|x_{j}-x_{k}\right|_{\mathbb{R}^{3}}}, \quad x=\left(x_{1}, \ldots, x_{d}\right) \in \mathbb{R}^{3 d},
$$

where $|\cdot|_{\mathbb{R}^{3}}$ denotes the Euclidean norm in $\mathbb{R}^{3}$. Moreover, the partial differential equation (3.1) is subject to asymptotic boundary conditions on the unbounded domain and an initial condition.

For practical applications that initiate the study of efficient and accurate numerical discretisations for (3.1) and more complex problems involving a time-dependent Hamiltonian, we refer to the works $[12,23,52,53]$. MCTDHF computations relevant to applications for the similar situation of a jellium are for instance reported in $[41,42]$.

It was shown in [29] that incorporation of a drift term modelling an intense laser pulse such that the linear operator is of the form $\mathscr{A}(t)=-\frac{1}{2}(\nabla+\mathrm{i} A(t))^{2}$ causes no additional difficulty. The reason is that the drift term introduces expressions of lower differentiation order due to $\nabla \ll \Delta$ [21], see also [29]. The corresponding analysis for electrons in an atom, where

$$
\mathscr{A}=-\frac{1}{2} \Delta-Z \sum_{j=1}^{d} \frac{1}{\left|x_{j}\right|_{\mathbb{R}^{3}}}
$$

was given in [24] for the second-order Strang splitting under the additional physical assumption that the electron density vanishes at the nucleus. However, this assumption cannot realistically be generalised to accommodate for high-order splitting methods studied in the present paper, as this would require the additional unphysical requirement that higher derivatives of the electron density vanish at the nucleus.

The MCTDHF approach, see also $[12,22,23,42,52,53]$, is closely related to the multi-configuration timedependent Hartree (MCTDH) approach in quantum molecular dynamics [7,8,11,36-38] and extends the timedependent Hartree-Fock method (TDHF) introduced in [15]. Similarly to low rank approximations of timedependent matrices, where a large system matrix is replaced by a linear combination of matrices of rank one, this model reduction makes high-dimensional Schrödinger equations such as (3.1) practicable for numerical simulations.

The MCTDHF approach for the time-dependent linear Schrödinger equation (3.1) relies on an approximation of the wave function by a linear combination of antisymmetrised products of functions, the orbitals, each depending on the coordinates of a single particle; further, the Schrödinger equation (3.1) is associated with an orthogonality condition on the tangent space of the approximation manifold. Via the Dirac-Frenkel timedependent variational principle $[15,16]$ the equations of motion for the orbitals and the coefficients in the linear combination of the products are deduced. Hence, the MCTDHF approach allows to replace the high-dimensional linear Schrödinger equation (3.1) by a system of coupled linear ordinary differential equations and nonlinear partial differential equations in three space dimensions, which is computationally treatable. 


\subsection{The MCTDHF equations}

The MCTDHF approach for time-dependent Schrödinger equations of the form (3.1) relies on an approximation of the multi-particle wave function by a linear combination of Hartree products

$$
\begin{gathered}
\Psi(x, t) \approx U(x, t)=\sum_{\mu \in M} a_{\mu}(t) \Phi_{\mu}(x, t), \quad x=\left(x_{1}, \ldots, x_{d}\right) \in \mathbb{R}^{3 d}, \quad 0 \leq t \leq T, \\
\Phi_{\mu}(x, \cdot)=\left(\bigotimes_{j=1}^{d} \phi_{\mu_{j}}\right)(x, \cdot)=\prod_{j=1}^{d} \phi_{\mu_{j}}\left(x_{j}, \cdot\right)=\phi_{\mu_{1}}\left(x_{1}, \cdot\right) \ldots \phi_{\mu_{d}}\left(x_{d}, \cdot\right), \\
M=\left\{\mu=\left(\mu_{1}, \ldots, \mu_{d}\right) \in \mathbb{N}^{d}: 1 \leq \mu_{j} \leq K, 1 \leq j \leq d\right\}
\end{gathered}
$$

for some $K \in \mathbb{N}$. In view of the Pauli exclusion principle [31], antisymmetry is imposed on (3.2a). That is, for any permutation $\sigma$ of $\{1, \ldots, d\}$, we require

$$
a_{\sigma(\mu)}=\operatorname{sign}(\sigma) a_{\mu}, \quad \sigma(\mu)=\left(\mu_{\sigma(1)}, \ldots, \mu_{\sigma(d)}\right), \quad \mu \in M,
$$

whence only $\left(\begin{array}{l}K \\ d\end{array}\right)$ different coefficients have to be determined in the actual computations; in particular it holds $a_{\mu}=a_{\left(\mu_{1}, \ldots, \mu_{d}\right)}=0$ whenever $\mu_{j}=\mu_{k}$ for some $1 \leq j<k \leq d$. For further details and an alternative representation of (3.2) by Slater determinants [45], Section 12-4, we refer to [29]. Slater determinants yield an alternative notation reflecting the antisymmetry in the coefficient tensor. This does not change the mathematical structure, however. In fact, when the equations of motion derived from the variational principle are propagated starting from an antisymmetric initial state, this property is preserved in the course of time integration, see [26].

In the following, we denote by $\phi=\left(\phi_{k}\right)_{1 \leq k \leq K}$ the vector of complex-valued orbitals, which depend on three spatial variables and on time, and by $a=\left(a_{\mu}\right)_{\mu \in M}$ the vector of time-dependent complex coefficients. For later use, we note that the relations

$$
\begin{aligned}
\partial_{t} U(x, t)= & \sum_{\mu \in M}\left(\frac{\mathrm{d}}{\mathrm{d} t} a_{\mu}(t) \Phi_{\mu}(x, t)+a_{\mu}(t) \partial_{t} \Phi_{\mu}(x, t)\right), \\
& \mathscr{A} U(x, t)=\sum_{\mu \in M} a_{\mu}(t) \mathscr{A} \Phi_{\mu}(x, t), \\
\partial_{t} \Phi_{\mu}(x, t)= & \sum_{\ell=1}^{d} \prod_{j=1}^{\ell-1} \phi_{\mu_{j}}\left(x_{j}, t\right) \partial_{t} \phi_{\mu_{\ell}}\left(x_{\ell}, t\right) \prod_{j=\ell+1}^{d} \phi_{\mu_{j}}\left(x_{j}, t\right), \\
\mathscr{A} \Phi_{\mu}(x, t)= & \sum_{\ell=1}^{d} \prod_{j=1}^{\ell-1} \phi_{\mu_{j}}\left(x_{j}, t\right) \mathscr{A}_{0} \phi_{\mu_{\ell}}\left(x_{\ell}, t\right) \prod_{j=\ell+1}^{d} \phi_{\mu_{j}}\left(x_{j}, t\right),
\end{aligned}
$$

where $\mathscr{A}_{0}=-\frac{1}{2} \Delta=-\frac{1}{2} \Delta_{\xi}$ involves the Laplacian $\Delta_{\xi}=\partial_{\xi_{1}}^{2}+\partial_{\xi_{2}}^{2}+\partial_{\xi_{3}}^{2}$ in three space variables $\xi=\left(\xi_{1}, \xi_{2}, \xi_{3}\right) \in$ $\mathbb{R}^{3}$, further imply

$$
\begin{array}{rl}
\mathrm{i} \partial_{t} & U(x, t)-\mathscr{H}(x) U(x, t) \\
=\sum_{\mu \in M} \mathrm{i} \frac{\mathrm{d}}{\mathrm{d} t} a_{\mu}(t) \Phi_{\mu}(x, t)+\sum_{\mu \in M} \sum_{\ell=1}^{d} a_{\mu}(t) \prod_{j=1}^{\ell-1} \phi_{\mu_{j}}\left(x_{j}, t\right)\left(\mathrm{i} \partial_{t} \phi_{\mu_{\ell}}\left(x_{\ell}, t\right)-\mathscr{A}_{0} \phi_{\mu_{\ell}}\left(x_{\ell}, t\right)\right) \prod_{j=\ell+1}^{d} \phi_{\mu_{j}}\left(x_{j}, t\right) \\
\quad-\sum_{\mu \in M} a_{\mu}(t) \mathscr{V}(x) \Phi_{\mu}(x, t), \quad x \in \mathbb{R}^{3 d}, \quad 0 \leq t \leq T,
\end{array}
$$

see also (3.1). 
Henceforth, we consider the set

$$
\mathscr{M}=\left\{V(b, \varphi)=\sum_{\mu \in M} b_{\mu} \bigotimes_{j=1}^{d} \varphi_{\mu_{j}}: b \in \mathbb{C}^{|M|}, \varphi \in\left(L^{2}\left(\mathbb{R}^{3}\right)\right)^{K}\right\} \subset L^{2}\left(\mathbb{R}^{3 d}\right),
$$

which has the structure of a manifold under a certain non-degeneracy condition, see (3.8) and an exhaustive discussion in [27]. The associated tangent space $\mathscr{T}_{V} \mathscr{M} \subset L^{2}\left(\mathbb{R}^{3 d}\right)$ at $V=V(b, \varphi) \in \mathscr{M}$ is given by

$$
\mathscr{T}_{V} \mathscr{M}=\left\{W(c, \chi)=\sum_{\mu \in M} c_{\mu} \bigotimes_{j=1}^{d} \varphi_{\mu_{j}}+\sum_{\mu \in M} \sum_{\ell=1}^{d} b_{\mu} \bigotimes_{j=1}^{\ell-1} \varphi_{\mu_{j}} \otimes \chi_{\mu_{\ell}} \otimes \bigotimes_{j=\ell+1}^{d} \varphi_{\mu_{j}}: c \in \mathbb{C}^{|M|}, \chi \in\left(L^{2}\left(\mathbb{R}^{3}\right)\right)^{K}\right\} .
$$

For the approximation of $(3.1)$, for any $U(\cdot, t) \in \mathscr{M}$, we require the time derivative $\partial_{t} U(\cdot, t)$ to be chosen such that the Galerkin condition

$$
\left(\delta U \mid \mathrm{i} \partial_{t} U(\cdot, t)-\mathscr{H} U(\cdot, t)\right)_{L^{2}\left(\mathbb{R}^{3 d}\right)}=0, \quad \delta U \in \mathscr{T}_{U(\cdot, t)} \mathscr{M}, \quad 0 \leq t \leq T,
$$

on the tangent space is satisfied; by means of (3.3), we thus obtain

$$
\begin{aligned}
& \sum_{\mu \in M} \mathrm{i} \frac{\mathrm{d}}{\mathrm{d} t} a_{\mu}(t)\left(\delta U \mid \Phi_{\mu}(\cdot, t)\right)_{L^{2}\left(\mathbb{R}^{3 d}\right)}+\sum_{\mu \in M} \sum_{\ell=1}^{d} a_{\mu}(t)\left(\delta U \mid\left(\bigotimes_{j=1}^{\ell-1} \phi_{\mu_{j}} \otimes\left(\mathrm{i} \partial_{t} \phi_{\mu_{\ell}}-\mathscr{A}_{0} \phi_{\mu_{\ell}}\right) \otimes \bigotimes_{j=\ell+1}^{d} \phi_{\mu_{j}}\right)(\cdot, t)\right)_{L^{2}\left(\mathbb{R}^{3 d}\right)} \\
& \quad-\sum_{\mu \in M} a_{\mu}(t)\left(\delta U|\mathscr{V}| \Phi_{\mu}(\cdot, t)\right)_{L^{2}\left(\mathbb{R}^{3 d}\right)}=0, \quad \delta U \in \mathscr{T}_{U(\cdot, t)} \mathscr{M}, \quad 0 \leq t \leq T .
\end{aligned}
$$

Furthermore, we impose the following orthogonality and gauge conditions on the orbitals

$$
\left(\phi_{k}(\cdot, t) \mid \phi_{\ell}(\cdot, t)\right)_{L^{2}\left(\mathbb{R}^{3}\right)}=\delta_{k \ell}, \quad\left(\phi_{k}(\cdot, t) \mid \mathrm{i} \partial_{t} \phi_{\ell}(\cdot, t)\right)_{L^{2}\left(\mathbb{R}^{3}\right)}=\left(\phi_{k}(\cdot, t)\left|\mathscr{A}_{0}\right| \phi_{\ell}(\cdot, t)\right)_{L^{2}\left(\mathbb{R}^{3}\right)}, \quad 0 \leq t \leq T, 1 \leq k, \ell \leq K,
$$

where $\delta_{k \ell}$ denotes the Kronecker-delta, i.e. $\delta_{k \ell}=1$ if $k=\ell$ and $\delta_{k \ell}=0$ otherwise. These serve to make the representation of the wave function and tangent space unique in the course of the evolution; note that $\mathscr{A}_{0}$ could be replaced by any self-adjoint operator yielding different equations of motion with the same mathematical properties, see $[7,27,36]$.

Consequently, via the Dirac-Frenkel time-dependent variational approximation principle $[15,16]$, a system of coupled ordinary and partial differential equations for the coefficients and the orbitals

$$
\begin{gathered}
\mathrm{i} \frac{\mathrm{d}}{\mathrm{d} t} a_{\mu}(t)=\sum_{\nu \in M}\left(\Phi_{\mu}(\cdot, t)|\mathscr{V}| \Phi_{\nu}(\cdot, t)\right)_{L^{2}\left(\mathbb{R}^{3 d}\right)} a_{\nu}(t), \\
\mathrm{i} \partial_{t} \phi_{k}(\xi, t)=\mathscr{A}_{0} \phi_{k}(\xi, t)+(I-P) \sum_{\ell, m=1}^{K} \rho_{k \ell}^{-1}(t)\left(\psi_{\ell}(\cdot, t)|\mathscr{V}| \psi_{m}(\cdot, t)\right)_{L^{2}\left(\mathbb{R}^{3(d-1)}\right)} \phi_{m}(\xi, t), \\
\xi \in \mathbb{R}^{3}, \quad 0 \leq t \leq T, \quad \mu \in M, \quad 1 \leq k \leq K,
\end{gathered}
$$

under asymptotic boundary conditions on the unbounded domain and certain initial conditions results. Here, the single-hole functions $\psi=\left(\psi_{k}\right)_{1 \leq k \leq K}$ are defined by

$$
\psi_{k}(\widetilde{x}, t)=\left(\phi_{k} \mid U(\cdot, \widetilde{x}, t)\right)_{L^{2}\left(\mathbb{R}^{3}\right)}, \quad \widetilde{x} \in \mathbb{R}^{3(d-1)}, \quad 0 \leq t \leq T, \quad 1 \leq k \leq K ;
$$

by the definition of the Hartree products (3.2) and the orthogonality relations (3.5) it follows

$$
\begin{gathered}
\psi_{k}(\widetilde{x}, t)=\sum_{\widetilde{\mu} \in \widetilde{M}} a_{(k, \widetilde{\mu})}(t) \prod_{j=2}^{d} \phi_{\mu_{j}}\left(x_{j}, t\right), \\
\widetilde{x}=\left(x_{2}, \ldots, x_{d}\right) \in \mathbb{R}^{3(d-1)}, \quad 0 \leq t \leq T, \quad 1 \leq k \leq K,
\end{gathered}
$$


with index set $\widetilde{M}=\left\{\widetilde{\mu}=\left(\mu_{2}, \ldots, \mu_{d}\right) \in \mathbb{N}^{d-1}: 1 \leq \mu_{j} \leq K, 2 \leq j \leq d\right\}$. The time-dependent density matrix $\rho=\left(\rho_{k \ell}\right)_{1 \leq k, \ell \leq K}$ with entries

$$
\rho_{k \ell}(t)=\left(\psi_{k}(\cdot, t) \mid \psi_{\ell}(\cdot, t)\right)_{L^{2}\left(\mathbb{R}^{3(d-1)}\right)}=\sum_{\widetilde{\mu} \in \widetilde{M}} \overline{a_{(k, \widetilde{\mu})}(t)} a_{(\ell, \widetilde{\mu})}(t), \quad 0 \leq t \leq T, \quad 1 \leq k, \ell \leq K,
$$

is required to be nonsingular; we denote by $\rho_{j m}^{-1}$ the entries of its inverse $\rho^{-1}$. Moreover, we denote by $P$ the orthogonal projector onto the linear space spanned by $\phi$; more precisely, we set

$$
P \chi(\xi, t)=\sum_{k=1}^{K}\left(\phi_{k}(\cdot, t) \mid \chi(\cdot, t)\right)_{L^{2}\left(\mathbb{R}^{3}\right)} \phi_{k}(\xi, t), \quad \xi \in \mathbb{R}^{3}, \quad 0 \leq t \leq T .
$$

We note that the inner products in (3.6) reduce to six- and three-dimensional integrals, respectively, since $\mathscr{V}$ is a sum of two-particle interactions only, see (3.1b); in particular, the right-hand sides of the differential equations in (3.6) comprise terms of the forms

$$
\iint_{\mathbb{R}^{3} \times \mathbb{R}^{3}} \frac{1}{|\xi-\eta|} \overline{f_{1}(\xi) f_{2}(\eta)} g_{1}(\xi) g_{2}(\eta) \mathrm{d} \xi \mathrm{d} \eta, \quad \int_{\mathbb{R}^{3}} \frac{1}{|\xi-\eta|} \overline{f_{1}(\xi)} g_{1}(\xi) \mathrm{d} \xi f_{2}(\eta), \quad \eta \in \mathbb{R}^{3} .
$$

Besides, it is noteworthy that in (3.6), due to the gauge condition (3.5), the single-particle operator $\mathscr{A}_{0}$ appears outside the projection in the partial differential equations for the orbitals. Choosing the initial conditions for the MCTDHF equations (3.6) such that $\rho(0)$ is nonsingular ensures that this also holds for $\rho(t)$ at any time $0 \leq t \leq T$, at least for $T>0$ sufficiently small; in [5,6,50], a sufficient condition for the invertibility of the density matrix, globally in time, is given. Under the assumption that a sufficiently regular analytical solution exists, a rigorous derivation of the MCTDHF equations is found in [7,37], see also [33] for a detailed discussion.

The following existence result for a unique regular solution of the MCTDHF equations (3.6)-(3.9), established in [29], is essential for our stability and convergence analysis.

Theorem 3.1 [29]. Assume that the initial conditions for the MCTDHF equations (3.6)-(3.9) are subject to the orthonormality constraints (3.5) and that the initial density matrix $\rho(0)$ is nonsingular. Assume further that the initial values for the orbitals satisfy $\phi(\cdot, 0) \in\left(H^{m}\left(\mathbb{R}^{3}\right)\right)^{K}$ for some $m \geq 2$. Then, there exists a unique strong solution of the MCTDHF equations such that $\phi(\cdot, t) \in\left(H^{m}\left(\mathbb{R}^{3}\right)\right)^{K}, 0 \leq t<T$, where either $T=\infty$ or $\rho(t)$ becomes singular for $t \uparrow T$. Moreover, the function $U$ defined in (3.2) satisfies $U(\cdot, t) \in H^{m}\left(\mathbb{R}^{3}\right)$ for $0 \leq t<T$ and solves the Dirac-Frenkel variational equation (3.4).

For the following considerations, it is useful to rewrite the MCTDHF equations (3.6)-(3.9), subject to asymptotic boundary conditions on the unbounded domain and certain initial conditions, as a nonlinear evolutionary problem of the form

$$
u^{\prime}(t)=F(u(t)), \quad 0 \leq t \leq T, \quad u(0) \text { given, }
$$

for $u(t)=(a(t), \phi(\cdot, t))^{T}$ comprising the coefficients and the orbitals (written as a column vector). The righthand side

$$
\begin{gathered}
F=-\mathrm{i}\left(B_{1}, \mathscr{A}_{0} \otimes I+B_{2}\right)^{T}, \quad \mathscr{A}_{0}=-\frac{1}{2} \Delta, \\
B_{1}(u(t))=\sum_{\nu \in M}\left(\Phi_{\mu}(\cdot, t)|\mathscr{V}| \Phi_{\nu}(\cdot, t)\right)_{L^{2}\left(\mathbb{R}^{3 d}\right)} a_{\nu}(t), \\
B_{2}(u(t))=(I-P) \sum_{\ell, m=1}^{K} \rho_{k \ell}^{-1}(t)\left(\psi_{\ell}(\cdot, t)|\mathscr{V}| \psi_{m}(\cdot, t)\right)_{L^{2}\left(\mathbb{R}^{3(d-1)}\right)} \phi_{m}(\xi, t),
\end{gathered}
$$

involves the Kronecker product of the linear differential operator $\mathscr{A}_{0}$ and the identity matrix of dimension $K$; the operator $B_{1}$, defined by the right-hand side of the ordinary differential equation for the coefficient vector, is linear in $a$ and further depends on the potential $\mathscr{V}$ and $\phi(\cdot, t)$; similarly, the nonlinear operator $B_{2}$ depends on $a, \phi(\cdot, t)$, and $\mathscr{V}$. 


\subsection{High-order variational splitting methods}

For the approximate solution of the time-dependent linear Schrödinger equation (3.1), we study high-order variational splitting methods that rely on a decomposition of the Hamiltonian in (3.1) into $\mathscr{H}=\mathscr{A}+\mathscr{V}$ and a suitable composition of the solutions to the variational subproblems

$$
\begin{aligned}
& \left(\delta U \mid \mathrm{i} \partial_{t} U(\cdot, t)-\mathscr{A} U(\cdot, t)\right)_{L^{2}\left(\mathbb{R}^{3 d}\right)}=0, \quad \delta U \in \mathscr{T}_{U(\cdot, t)} \mathscr{M}, \quad 0 \leq t \leq T, \\
& \left(\delta U \mid \mathrm{i} \partial_{t} U(\cdot, t)-\mathscr{V} U(\cdot, t)\right)_{L^{2}\left(\mathbb{R}^{3 d}\right)}=0, \quad \delta U \in \mathscr{T}_{U(\cdot, t)} \mathscr{M}, \quad 0 \leq t \leq T ;
\end{aligned}
$$

more precisely, we use the fact that for some given initial value $U(\cdot, t) \in \mathscr{M}$ the (numerical) solutions to (3.12) at time $t+\Delta t, 0 \leq t \leq t+\Delta t \leq T$, are available. This method was first introduced based on the coefficients for the second-order Strang splitting in [32]; the variational formulation of the subproblems arising in the splitting motivates the denotation as variational splitting.

In regard to a theoretical analysis and the numerical realisation of variational splittings, it is essential that the variational problems (3.12) are equivalent to differential equations that arise when applying an exponential operator splitting method to the MCTDHF equations (3.6)-(3.9), see also (3.10). Namely, due to the fact that $\partial_{t} U(\cdot, t), \mathscr{A} U(\cdot, t) \in \mathscr{T}_{U(\cdot, t)} \mathscr{M}$ for all $U(\cdot, t) \in \mathscr{M} \cap H^{2}\left(\mathbb{R}^{3 d}\right)$, the first subproblem in (3.12) corresponds to the solution of the free Schrödinger equation i $\partial_{t} U=\mathscr{A} U$; for initial data in $\mathscr{M}$ this problem decouples into $\frac{\mathrm{d}}{\mathrm{d} t} a=0$ and the single-particle free Schrödinger equations $\mathrm{i} \partial_{t} \phi_{k}=\mathscr{A}_{0} \phi_{k}, 1 \leq k \leq K$, solvable by Fast Fourier techniques. On the other hand, the second subproblem leads to a nonlinear system of coupled differential equations for the coefficients $\mathrm{i} \frac{\mathrm{d}}{\mathrm{d} t} a=B_{1}(a, \phi)$ and for the orbitals $\mathrm{i} \partial_{t} \phi=B_{2}(a, \phi)$ that in practice is solved by an explicit time integration method together with an occasional reorthogonalisation of the orbitals. If suitably chosen, this approximation does not affect the order of the overall method, see [32], where additionally the efficiency and accuracy of several schemes from the literature are compared. Hence, variational splitting methods for (3.1) are associated with exponential operator splitting methods for the following evolutionary problem with operators $\mathscr{A}_{0}, B_{1}, B_{2}$ given in (3.11)

$$
\begin{gathered}
u^{\prime}(t)=A u(t)+B(u(t)), \quad 0 \leq t \leq T, \quad u(0) \text { given}, \\
A=-\mathrm{i}\left(\begin{array}{c}
0 \\
\mathscr{A}_{0} \otimes I
\end{array}\right), \quad B=-\mathrm{i}\left(\begin{array}{c}
B_{1} \\
B_{2}
\end{array}\right)
\end{gathered}
$$

and the efficient numerical solution of the subproblems

$$
\begin{array}{cc}
u^{\prime}(t)=A u(t), & 0 \leq t \leq T, \quad u(0) \text { given } \\
u^{\prime}(t)=B(u(t)), & 0 \leq t \leq T, \quad u(0) \text { given, }
\end{array}
$$

see also (3.6), (3.10), and (3.12).

\subsection{Convergence analysis}

In this section, we specify our main result on the convergence behaviour of high-order exponential operator splitting methods (2.2) for the time integration of the MCTDHF equations (3.6)-(3.9), written in the abstract form (3.13). We recall that Theorem 3.1 states necessary conditions for the existence and required regularity of the exact solution of the MCTDHF equations (3.6)-(3.9). Namely, it holds

$$
u(t)=(a(t), \phi(\cdot, t))^{T} \in \mathscr{X}_{m}=\mathbb{C}^{|M|} \times\left(H^{m}\left(\mathbb{R}^{3}\right)\right)^{K}, \quad 0 \leq t \leq T,
$$


for some $m \geq 0$ and $T>0$, provided that the initial value $u(0)$ is suitably chosen. Clearly, the linear space $\mathscr{X}_{m}$ forms a Banach space with associated norm

$$
\begin{gathered}
\|v\|_{\mathscr{X}_{m}}=\max \left\{|z|_{\mathbb{C}|M|},\|\chi\|_{\left(H^{m}\left(\mathbb{R}^{3}\right)\right)^{K}}\right\}, \quad v=(z, \chi) \in \mathscr{X}_{m}, \\
|z|_{\mathbb{C}^{|M|} \mid}=\left(\sum_{\mu \in M}\left|z_{\mu}\right|^{2}\right)^{1 / 2}, \quad z=\left(z_{\mu}\right)_{\mu \in M} \in \mathbb{C}^{|M|}, \\
\|\chi\|_{\left(H^{m}\left(\mathbb{R}^{3}\right)\right)^{K}}=\max _{1 \leq k \leq K}\left\|\chi_{k}\right\|_{H^{m}\left(\mathbb{R}^{3}\right)}, \quad \chi=\left(\chi_{k}\right)_{1 \leq k \leq K} \in\left(H^{m}\left(\mathbb{R}^{3}\right)\right)^{K} .
\end{gathered}
$$

Theorem 3.2. Suppose that the MCTDHF equations (3.6)-(3.9), written as an abstract evolutionary problem (3.13), possess a uniquely determined sufficiently regular solution $u$ on the time interval $[0, T]$ and that the associated density matrix $\rho(t)$ is nonsingular for $0 \leq t \leq T$. Then, for any exponential operator splitting method (2.2) of (nonstiff) order $p \geq 1$, the following error estimates are valid.

(i) Provided that $\|u(t)\|_{\mathscr{X}_{m}} \leq M_{m}$ for $0 \leq t \leq T$ with $m=p=2$ or $m=2 p-3$ for $p \geq 3$, respectively, the bound

$$
\left\|u_{n}-u\left(t_{n}\right)\right\|_{\mathscr{X}_{0}} \leq C h^{p}, \quad 0 \leq n \leq N, \quad t_{N} \leq T
$$

holds true with constant $C$ depending on $M_{m}$.

(ii) Provided that $\left\|u\left(t_{n}\right)\right\|_{\mathscr{X}_{m}} \leq M_{m}$ for $0 \leq t \leq T$ with $m=p=2,3$ or $m=2 p-4$ for $p \geq 4$, respectively, the bound

$$
\left\|u_{n}-u\left(t_{n}\right)\right\|_{\mathscr{X}_{1}} \leq C h^{p-1}, \quad 0 \leq n \leq N, \quad t_{N} \leq T,
$$

holds true with constant $C$ depending on $M_{m}$.

Proof. Our convergence proof generalises the error analysis of [29], where the second-order Strang type splitting method (2.3) is studied for the MCTDHF equations (3.6)-(3.9), in abstract form written as (3.13), to highorder schemes (2.2), see also [34]. In order to ensure the stability of the subproblem for the nonlinear operator $B$ involving the potential $\mathscr{V}$, first the error estimate with respect to the $\mathscr{X}_{1}$ norm has to be established; then, the bound in $\mathscr{X}_{0}$ is obtained. The main steps of the proof are given below; for simplicity, we may assume that the first component of any element $v=(z, \chi) \in \mathscr{X}_{m}$ is of unit norm.

Step 1. Stability in $\mathscr{X}_{1}$. If

$$
\|v\|_{\mathscr{X}_{m}} \leq M_{m}, \quad\|w\|_{\mathscr{X}_{m}} \leq M_{m}, \quad v, w \in \mathscr{X}_{m}
$$

then we have

$$
\begin{aligned}
& \left\|\mathrm{e}^{t D_{A}} v\right\|_{\mathscr{X}_{m}}=\|v\|_{\mathscr{X}_{m}}, \quad v \in \mathscr{X}_{m}, \quad t \geq 0, \quad m \geq 0 \\
& \left\|B^{\prime}(v)\right\|_{\mathscr{X}_{m} \leftarrow \mathscr{X}_{m}} \leq C\|v\|_{\mathscr{X}_{m}}, \quad v \in \mathscr{X}_{m}, \quad 0 \leq t \leq T, \quad m \geq 1, \\
& \left\|\mathrm{e}^{t D_{B}} v-\mathrm{e}^{t D_{B}} w\right\|_{\mathscr{X}_{m}} \leq \mathrm{e}^{c t}\|v-w\|_{\mathscr{X}_{m}}, \quad v, w \in \mathscr{X}_{m}, \quad 0 \leq t \leq T, \quad m \geq 1,
\end{aligned}
$$

where $C$ and $c$ depend on $M_{m}$, see [29]. For any high-order exponential operator splitting method (2.2) involving several compositions of the subproblems in $(3.13 \mathrm{~b})$, a stability bound with respect to the norm of $\mathscr{X}_{1}$ is a direct consequence of the above bounds; namely, it follows

$$
\left\|\mathscr{S}\left(h_{n}, v\right)-\mathscr{S}\left(h_{n}, w\right)\right\|_{\mathscr{X}_{1}} \leq \mathrm{e}^{c_{1} h_{n}}\|v-w\|_{\mathscr{X}_{1}}, \quad 0 \leq n \leq N-1,
$$

with $c_{1}$ depending on $M_{1}$.

Step 2. Local error estimate in $\mathscr{X}_{1}$. Assuming that $v \in \mathscr{X}_{0}$ satisfies the additional regularity requirement

$$
\|v\|_{\mathscr{X}_{m}} \leq M_{m}, \quad v \in \mathscr{X}_{m}
$$


with $m=p=2,3$ or $m=2 p-4$ for $p \geq 4$, respectively, by means of the local error expansion given in Theorem 2.1 and the bound for the iterated Lie-commutator $\operatorname{ad}_{D_{A}}^{p-1}\left(D_{B}\right)$ in $\mathscr{X}_{1}$ given in Lemma 3.4, the estimate

$$
\left\|\mathscr{S}\left(h_{n}, v\right)-\mathscr{E}\left(h_{n}, v\right)\right\|_{\mathscr{X}_{1}} \leq C_{1} h_{n}^{p}, \quad 0 \leq n \leq N-1,
$$

follows with constant $C_{1}$ depending on $M_{m}$.

Step 3. Global error estimate in $\mathscr{X}_{1}$. Combining the above stability and local error estimate, a standard Lady Windermere's fan argument [19], Section II.3 yields the convergence bound (3.16); in particular, if

$$
\left\|u_{0}\right\|_{\mathscr{X}_{m}} \leq M_{m}, \quad u_{0} \in \mathscr{X}_{m},
$$

with $m=p=2,3$ or $m=2 p-4$ for $p \geq 4$, respectively, the boundedness of the splitting solution

$$
\left\|u_{n}\right\|_{\mathscr{X}_{1}} \leq C, \quad 0 \leq t_{n} \leq T,
$$

follows with constant $C$ depending on $M_{m}$.

Step 4. $\mathscr{X}_{1}$-conditional stability in $\mathscr{X}_{0}$. If

$$
\|v\|_{\mathscr{X}_{1}} \leq M_{1}, \quad\|w\|_{\mathscr{X}_{1}} \leq M_{1}, \quad v, w \in \mathscr{X}_{1}
$$

the estimate

$$
\left\|\mathscr{S}\left(h_{n}, v\right)-\mathscr{S}\left(h_{n}, w\right)\right\|_{\mathscr{X}_{0}} \leq \mathrm{e}^{c_{0} h_{n}}\|v-w\|_{\mathscr{X}_{0}}, \quad 0 \leq n \leq N-1,
$$

holds with $c_{0}$ depending on $M_{1}$, see also [29].

Step 5. Local error estimate in $\mathscr{X}_{0}$. If

$$
\|v\|_{\mathscr{X}_{m}} \leq M_{m}, \quad v \in \mathscr{X}_{m}
$$

with $m=p=2$ or $m=2 p-3$ for $p \geq 3$, the local error expansion of Theorem 2.1 together with the commutator bound of Lemma 3.4 with respect to the $\mathscr{X}_{0}$ norm yields

$$
\left\|\mathscr{S}\left(h_{n}, v\right)-\mathscr{E}\left(h_{n}, v\right)\right\|_{\mathscr{X}_{0}} \leq C_{0} h_{n}^{p+1}, \quad 0 \leq n \leq N-1,
$$

where $C_{0}$ depends on $M_{m}$.

Step 6. Global error estimate in $\mathscr{X}_{0}$. Finally, making use of the fact that the splitting solution remains bounded in $\mathscr{X}_{1}$, see Step 3 , the stability estimate and the local error bound with respect to the $\mathscr{X}_{0}$ norm imply the desired convergence estimate of order $p$ in $\mathscr{X}_{0}$.

Remark 3.3. It is a well-known phenomenon that the lack of regularity in the data will affect the attainable convergence order of a numerical method. For instance, in the situation of Theorem 3.2, if $\|u(t)\| \mathscr{K}_{k} \leq M_{k}$ (but not $\left.\|u(t)\|_{\mathscr{X}_{k+1}} \leq M_{k+1}\right)$ for $0 \leq t \leq T$ with $q=k=2$ or $q=\frac{k+3}{2} \in \mathbb{N}, q \geq 3$, respectively, the error estimate

$$
\left\|u_{n}-u(t)\right\|_{\mathscr{X}_{0}} \leq C h^{q}, \quad 0 \leq n \leq N, \quad t_{N} \leq T,
$$

follows; see also [48] for a theoretical result and a numerical illustration of the encountered order reduction for linear Schrödinger equations.

\subsection{Commutator bounds}

In the following, we derive bounds for the iterated Lie-commutators of the operators arising in the MCTDHF equations (3.6)-(3.9); we recall the abstract formulation (3.13) and definition (A.3j)-(A.3k).

Lemma 3.4. The operators $A$ and $B$ associated with the MCTDHF equations (3.6)-(3.9) satisfy the iterated commutator bounds

$$
\begin{gathered}
\left\|\operatorname{ad}_{D_{A}}^{2}\left(D_{B}\right) v\right\|_{\mathscr{X}_{1}} \leq C\left(\|v\|_{\mathscr{X}_{3}}\right), \\
\left\|\operatorname{ad}_{D_{A}}^{j+2}\left(D_{B}\right) v\right\|_{\mathscr{X}_{k}} \leq C\left(\|v\|_{\mathscr{X}_{k+2 j+1}}\right), \quad j \geq 1, \quad k=0,1,
\end{gathered}
$$

where the quantity $C$ depends on the respective norm in a nonlinear way. 
Proof. In [29], it was shown that the second iterated Lie-commutator $\operatorname{ad}_{D_{A}}^{2}\left(D_{B}\right)$ involving the Lie derivatives of the linear operator $A$ and the nonlinear operator $B$, defined by the right-hand side of the MCTDHF equations (3.6)-(3.9), see (3.13), comprises terms of the form $\overline{f_{1}} f_{2} \Delta f_{3}$ and $f_{3} \overline{\nabla f_{1}} \cdot \nabla f_{2}$, respectively, where $f_{1}, f_{2}, f_{3}$ are any orbitals; for a complex-valued function in three spatial variables $\eta=\left(\eta_{1}, \eta_{2}, \eta_{3}\right) \in \mathbb{R}^{3}$, we denote by $\nabla=\left(\partial_{\eta_{1}}, \partial_{\eta_{2}}, \partial_{\eta_{3}}\right)$ the gradient operator and by $\Delta=\nabla \cdot \nabla=\partial_{\eta_{1}}^{2}+\partial_{\eta_{2}}^{2}+\partial_{\eta_{3}}^{2}$ the corresponding Laplacian with respect to $\eta$. As a consequence, by means of the Hölder and Sobolev inequalities

$$
\begin{gathered}
\left\|f_{1} f_{2}\right\|_{L^{2}\left(\mathbb{R}^{3}\right)} \leq\left\|f_{1}\right\|_{L^{\infty}\left(\mathbb{R}^{3}\right)}\left\|f_{2}\right\|_{L^{2}\left(\mathbb{R}^{3}\right)} \leq C\left\|f_{1}\right\|_{H^{2}\left(\mathbb{R}^{3}\right)}\left\|f_{2}\right\|_{L^{2}\left(\mathbb{R}^{3}\right)}, \\
\left\|f_{1} f_{2}\right\|_{L^{2}\left(\mathbb{R}^{3}\right)} \leq C\left\|f_{1}\right\|_{L^{4}\left(\mathbb{R}^{3}\right)}\left\|f_{2}\right\|_{L^{4}\left(\mathbb{R}^{3}\right)} \leq C\left\|f_{1}\right\|_{H^{1}\left(\mathbb{R}^{3}\right)}\left\|f_{2}\right\|_{H^{1}\left(\mathbb{R}^{3}\right)}, \\
\left\|f_{1} f_{2}\right\|_{H^{2}\left(\mathbb{R}^{3}\right)} \leq C\left\|f_{1}\right\|_{H^{2}\left(\mathbb{R}^{3}\right)}\left\|f_{2}\right\|_{H^{2}\left(\mathbb{R}^{3}\right)}, \\
\left\|f_{1} f_{2} f_{3}\right\|_{L^{2}\left(\mathbb{R}^{3}\right)} \leq C\left\|f_{1}\right\|_{L^{6}\left(\mathbb{R}^{3}\right)}\left\|f_{2}\right\|_{L^{6}\left(\mathbb{R}^{3}\right)}\left\|f_{3}\right\|_{L^{6}\left(\mathbb{R}^{3}\right)} \leq C\left\|f_{1}\right\|_{H^{1}\left(\mathbb{R}^{3}\right)}\left\|f_{2}\right\|_{H^{1}\left(\mathbb{R}^{3}\right)}\left\|f_{3}\right\|_{H^{1}\left(\mathbb{R}^{3}\right)},
\end{gathered}
$$

see also $[2,10,20,39]$, the following relations

$$
\begin{aligned}
\left\|\overline{f_{1}} f_{2} \Delta f_{3}\right\|_{L^{2}\left(\mathbb{R}^{3}\right)} & \leq C\left\|\overline{f_{1}} f_{2}\right\|_{H^{2}\left(\mathbb{R}^{3}\right)}\left\|\Delta f_{3}\right\|_{L^{2}\left(\mathbb{R}^{3}\right)} \\
& \leq C\left\|f_{1}\right\|_{H^{2}\left(\mathbb{R}^{3}\right)}\left\|f_{2}\right\|_{H^{2}\left(\mathbb{R}^{3}\right)}\left\|f_{3}\right\|_{H^{2}\left(\mathbb{R}^{3}\right)}, \\
\left\|f_{3} \overline{\nabla f_{1}} \cdot \nabla f_{2}\right\|_{L^{2}\left(\mathbb{R}^{3}\right)} & \leq C\left\|f_{3}\right\|_{H^{1}\left(\mathbb{R}^{3}\right)}\left\|\nabla f_{1}\right\|_{H^{1}\left(\mathbb{R}^{3}\right)}\left\|\nabla f_{2}\right\|_{H^{1}\left(\mathbb{R}^{3}\right)} \\
& \leq C\left\|f_{1}\right\|_{H^{2}\left(\mathbb{R}^{3}\right)}\left\|f_{2}\right\|_{H^{2}\left(\mathbb{R}^{3}\right)}\left\|f_{3}\right\|_{H^{2}\left(\mathbb{R}^{3}\right)},
\end{aligned}
$$

are obtained, which further imply the bounds

$$
\left\|\operatorname{ad}_{D_{A}}^{2}\left(D_{B}\right) v\right\|_{\mathscr{X}_{0}} \leq C\left(\|v\|_{\mathscr{X}_{2}}\right), \quad\left\|\operatorname{ad}_{D_{A}}^{2}\left(D_{B}\right) v\right\|_{\mathscr{X}_{1}} \leq C\left(\|v\|_{\mathscr{X}_{3}}\right) .
$$

In order to determine $\operatorname{ad}_{D_{A}}^{j}\left(D_{B}\right)$ for $j \geq 3$, it suffices to consider iterated Lie-commutators of the linear differential operator $A_{0}$ and the nonlinear multiplication operator $B_{0}$, defined by

$$
\begin{gathered}
A_{0}: D\left(A_{0}\right) \subset\left(L^{2}\left(\mathbb{R}^{3}\right)\right)^{3} \longrightarrow\left(L^{2}\left(\mathbb{R}^{3}\right)\right)^{3}: f=\left(f_{1}, f_{2}, f_{3}\right)^{T} \longmapsto \mathrm{i}\left(\Delta f_{1}, \Delta f_{2}, \Delta f_{3}\right)^{T}, \\
B_{0}: D\left(B_{0}\right) \subset\left(L^{2}\left(\mathbb{R}^{3}\right)\right)^{3} \longrightarrow L^{2}\left(\mathbb{R}^{3}\right): f=\left(f_{1}, f_{2}, f_{3}\right)^{T} \longmapsto \overline{f_{1}} f_{2} f_{3},
\end{gathered}
$$

with Fréchet-derivatives given by $A_{0}^{\prime}(f)=\mathrm{i} \Delta$ and $B_{0}^{\prime}(f)=\left(f_{2} f_{3} \overline{(\cdot)}, \overline{f_{1}} f_{3}(\cdot), \overline{f_{1}} f_{2}(\cdot)\right)$; due to $\Delta\left(\overline{f_{1}} f_{2} f_{3}\right)=$ $f_{2} f_{3} \overline{\Delta f_{1}}+\overline{f_{1}} f_{3} \Delta f_{2}+\overline{f_{1}} f_{2} \Delta f_{3}+2 f_{3} \overline{\nabla f_{1}} \cdot \nabla f_{2}+2 f_{2} \overline{\nabla f_{1}} \cdot \nabla f_{3}+2 \overline{f_{1}} \nabla f_{2} \cdot \nabla f_{3}$ we have

$$
\begin{aligned}
{\left[A_{0}, B_{0}\right](f) } & =A_{0}^{\prime}(f) B_{0}(f)-B_{0}^{\prime}(f) A_{0}(f)=\mathrm{i} \Delta\left(\overline{f_{1}} f_{2} f_{3}\right)-\left(f_{2} f_{3} \overline{\mathrm{i} \Delta f_{1}}+\overline{f_{1}} f_{3} \mathrm{i} \Delta f_{2}+\overline{f_{1}} f_{2} \mathrm{i} \Delta f_{3}\right) \\
& =2 \mathrm{i}\left(f_{2} f_{3} \overline{\Delta f_{1}}+f_{3} \overline{\nabla f_{1}} \cdot \nabla f_{2}+f_{2} \overline{\nabla f_{1}} \cdot \nabla f_{3}+\overline{f_{1}} \nabla f_{2} \cdot \nabla f_{3}\right),
\end{aligned}
$$

which shows that $\operatorname{ad}_{D_{A_{0}}}\left(D_{B_{0}}\right)=-\left[A_{0}, B_{0}\right]$ has a similar structure as $B_{0}$. Applying the previously given bounds yields

$$
\left\|\operatorname{ad}_{A_{0}}\left(B_{0}\right) f\right\|_{L^{2}\left(\mathbb{R}^{3}\right)} \leq C \max _{1 \leq \ell \leq 3}\left\|f_{\ell}\right\|_{H^{2}\left(\mathbb{R}^{3}\right)}, \quad\left\|\operatorname{ad}_{A_{0}}\left(B_{0}\right) f\right\|_{H^{1}\left(\mathbb{R}^{3}\right)} \leq C \max _{1 \leq \ell \leq 3}\left\|f_{\ell}\right\|_{H^{3}\left(\mathbb{R}^{3}\right)} .
$$

From the above considerations, we further obtain

$$
\left\|\operatorname{ad}_{D_{A}}^{3}\left(D_{B}\right) v\right\|_{\mathscr{X}_{0}} \leq C\left(\|v\|_{\mathscr{X}_{3}}\right), \quad\left\|\operatorname{ad}_{D_{A}}^{3}\left(D_{B}\right) v\right\|_{\mathscr{X}_{1}} \leq C\left(\|v\|_{\mathscr{X}_{4}}\right) ;
$$

by induction, the statement of Lemma 3.4 results.

\subsection{The cubic Schrödinger equation}

With the techniques presented above it is also possible to analyse high-order splitting methods for the cubic nonlinear Schrödinger equation

$$
\mathrm{i} \partial_{t} \psi(x, t)=-\frac{1}{2} \Delta \psi+\kappa|\psi(x, t)|^{2} \psi(x, t),
$$


where $\kappa>0$ and thus the solution shows a dissipative behaviour and exists globally in time. Note that if the model is modified such that $\kappa<0$, the solution generically becomes unbounded in finite time [47], thus restricting the applicability of our result. The operators (up to scalings) in the associated abstract problem (2.1)

$$
A: D(A) \subset L^{2}\left(\mathbb{R}^{3}\right) \longrightarrow L^{2}\left(\mathbb{R}^{3}\right): u \longmapsto \mathrm{i} \Delta u, \quad B: D(B) \subset L^{2}\left(\mathbb{R}^{3}\right) \longrightarrow L^{2}(\mathbb{R}): u \longmapsto|u|^{2} u,
$$

are closely related to the operators $A_{0}, B_{0}$ from (3.20). Thus, the iterated Lie-commutators can be computed analogously to (3.21), for instance,

$$
[A, B](u)=\mathrm{i} \Delta\left(|u|^{2} u\right)-\left(u^{2} \overline{\mathrm{i} \Delta u}+2|u|^{2} \mathrm{i} \Delta u\right)=2 \mathrm{i}\left(u^{2} \overline{\Delta u}+2 u \overline{\nabla u} \cdot \nabla u+\bar{u} \nabla u \cdot \nabla u\right) .
$$

The stability analysis uses (3.19), and thus the nonlinear term satisfies a Lipschitz condition in $H^{2}$, but not in $H^{1}$ or $L^{2}$. Consequently, for the derivation of a convergence result for (3.22), a three-stage procedure has to be applied, where in the first step convergence is studied in $H^{2}$ and subsequently the results in $H^{1}$ and $L^{2}$ are concluded with conditional stability estimates depending on the boundedness of the numerical solution in $H^{2}$, which was established in the first step. The analysis reflecting this fact was first given for the second-order Strang splitting in [34] and extended to time and space discretisations based on pseudo-spectral and high-order splitting methods in [49]. We formulate the result for high-order splitting methods in the following theorem:

Theorem 3.5. Suppose that the cubic nonlinear Schrödinger equation (3.22), written as an abstract evolutionary problem (3.13), possesses a uniquely determined sufficiently regular solution $u$ on the time interval $[0, T]$. Then, for any exponential operator splitting method (2.2) of (nonstiff) order $p \geq 2$, the following error estimates are valid.

(i) Provided that $\|u(t)\|_{H^{2 p}} \leq M_{2 p}$ for $0 \leq t \leq T$, the bound

$$
\left\|u_{n}-u\left(t_{n}\right)\right\|_{L^{2}} \leq C h^{p}, \quad 0 \leq n \leq N, \quad t_{N} \leq T,
$$

holds true with constant $C$ depending on $M_{2 p}$.

(ii) Provided that $\|u(t)\|_{H^{2 p-1}} \leq M_{2 p-1}$ for $0 \leq t \leq T$, the bound

$$
\left\|u_{n}-u\left(t_{n}\right)\right\|_{H^{1}} \leq C h^{p-1}, \quad 0 \leq n \leq N, \quad t_{N} \leq T,
$$

holds true with constant $C$ depending on $M_{2 p-1}$.

(iii) Provided that $\|u(t)\|_{H^{2 p-2}} \leq M_{2 p-2}$ for $0 \leq t \leq T$, the bound

$$
\left\|u_{n}-u\left(t_{n}\right)\right\|_{H^{2}} \leq C h^{p-2}, \quad 0 \leq n \leq N, \quad t_{N} \leq T,
$$

holds true with constant $C$ depending on $M_{2 p-2}$.

In order to illustrate the above convergence result, we consider the cubic Schrödinger equation (3.22) with $\kappa=1$ and localised initial condition

$$
\psi(x, 0)=\varrho(x) \mathrm{e}^{-\mathrm{i} \sigma(x)}, \quad \varrho(x)=\mathrm{e}^{-\left(x_{1}^{2}+x_{2}^{2}+x_{3}^{2}\right)}, \quad \sigma(x)=-\ln \left(\mathrm{e}^{x_{1}+x_{2}+x_{3}}+\mathrm{e}^{-x_{1}-x_{2}-x_{3}}\right), \quad x \in \mathbb{R}^{3} .
$$

For the time integration we apply different splitting methods involving $s$ stages of (nonstiff) order $p=s$ including the well-known first-order Lie-Trotter, second-order Strang, and fourth-order Yoshida splitting methods, see for example [18]. The problem is discretised in space by means of the Fourier pseudo-spectral method with $M=32$ basis functions in each space direction, see for instance [49] for details on the implementation. A numerical reference solution is computed by means of an optimised fourth-order splitting method constructed in [9]. In Figure 1, we display the global errors versus the time stepsizes, making use of the fact that the solution of the nonlinear subproblem

$$
\mathrm{i} \partial_{t} \psi(x, t)=\kappa|\psi(x, t)|^{2} \psi(x, t), \quad \psi(x, t)=\mathrm{e}^{-\mathrm{i} \kappa t|\psi(x, 0)|^{2}} \psi(x, 0),
$$



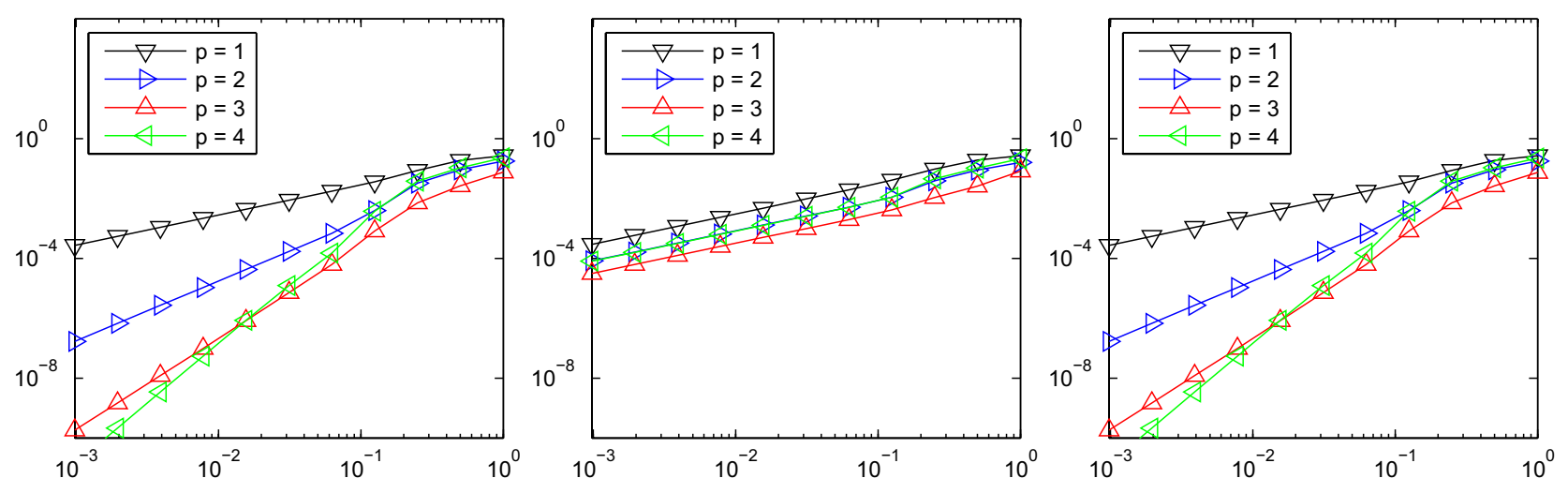

Figure 1. Global error of different splitting methods applied to the cubic Schrödinger equation (left). Corresponding results obtained for a numerical approximation of the nonlinear subproblem (3.27) by means of the explicit Euler method (middle) and a fourth-order explicit Runge-Kutta method (right).

can be computed with high accuracy by pointwise multiplication. In view of nonlinear Schrödinger equations such as the MCTDHF equations, where only numerical approximations to the solutions of the associated subproblems are available, we further apply the first-order explicit Euler method as well as the classical explicit Runge-Kutta method of order four for the approximation of the nonlinear subproblem. In the first case, for all splitting methods the order reduces to $p=1$, whereas in the latter case the computed approximation is sufficiently accurate in order to retain the (non)stiff orders of the considered splitting methods.

\section{Conclusions}

In the present work, we were concerned with the theoretical investigation of the convergence behaviour of high-order exponential operator splitting methods for nonlinear evolutionary Schrödinger equations. An essential tool in our error analysis, which relies on the framework of abstract evolution equations on Banach spaces and the formal calculus of Lie derivatives, is an expansion of the local error that remains suitable in the presence of unbounded nonlinear operators. In order to substantiate the general approach, we studied the MCTDHF equations, which constitute a system of coupled linear ordinary differential equations and low dimensional nonlinear partial differential equations. Utilising bounds for iterated Lie-commutators of the involved operators, we deduced a convergence estimate in $L^{2}$ under optimal regularity requirements on the analytical solution. The theoretical analysis was also particularized to the cubic Schrödinger equation and illustrated numerically.

In this work, the focus of attention was on theoretical aspects of time integration methods for nonlinear evolution equations, and we refrained from confirming the convergence result for the MCTDHF equations by a numerical example. The complexity of this application makes the implementation of time and space discretisations a highly difficult and involved task. Also, it is expected that the chosen spatial discretisation is critical for the observed convergence behaviour. In order to experimentally observe the theoretical bounds a sufficiently high resolution in space is needed which pushes computing recources to their limit, in particular for involved problems like the MCTDHF equations for a larger number of electrons. As a first step towards a better understanding of these questions, we intend to study full discretisations based on time-splitting finite element approximations [1] for related problems such as the Schrödinger-Poisson equation.

As mentioned in the introduction, numerical experiments for Gross-Pitaevskii systems have shown the favourable accuracy and efficiency of full discretisations based on pseudo-spectral methods in space and higherorder exponential operator splitting methods in time. However, it remains to back the numerical observations by a theoretical analysis. The investigation of these aspects is the subject of current [49] and future work. In 
particular, we plan to extend the presented stability and convergence analysis to other classes of nonlinear evolutionary Schrödinger equations such as Gross-Pitaevskii equations with additional rotation term.

Acknowledgements. We acknowledge the financial support by the Austrian Academy of Sciences' APART program and by the Austrian Science Fund (FWF) under projects P21620-N13 and P24157-N13.

\section{A. Appendix calculus of Lie derivatives}

A most useful tool in the statement and the theoretical error analysis of high-order exponential operator splitting methods for nonlinear evolution equations is the formal calculus of Lie derivatives, which is suggestive of the less involved linear case, see also [18]. In the following, we review basic definitions and results needed in the derivation of our local error expansion; we note that the calculus of Lie derivatives is used as a formal means under the tacit requirement that the arising unbounded operators and compositions thereof are well-defined on suitably chosen domains and time intervals.

\section{A.1. Evolution operator and Lie derivative}

We consider an initial value problem of the form

$$
u^{\prime}(t)=F(u(t)), \quad 0 \leq t \leq T, \quad u(0)=u_{0},
$$

where the unbounded nonlinear operator $F: D(F) \subset X \rightarrow X$ is defined on a non-empty subset of the underlying Banach space $\left(X,\|\cdot\|_{X}\right)$. Formally, the exact solution of the evolutionary problem (A.1) is given by

$$
u(t)=\mathscr{E}_{F}\left(t, u_{0}\right), \quad 0 \leq t \leq T,
$$

with the evolution operator $\mathscr{E}_{F}$ depending on the actual time and the initial value; as we restrict ourselves to an autonomous differential equation, we may omit the dependence on the initial time. Furthermore, it is most helpful to employ the formal notation

$$
u(t)=\mathrm{e}^{t D_{F}} u_{0}, \quad 0 \leq t \leq T .
$$

More precisely, the evolution operator $\left(\mathrm{e}^{t D_{F}}\right)_{0 \leq t \leq T}$ and the Lie derivative $D_{F}$ associated with $F$ are given through the relations

$$
\mathrm{e}^{t D_{F}} G u_{0}=G\left(\mathscr{E}_{F}\left(t, u_{0}\right)\right), \quad 0 \leq t \leq T, \quad D_{F} G u_{0}=G^{\prime}\left(u_{0}\right) F\left(u_{0}\right),
$$

for any unbounded nonlinear operator $G: D(G) \subset X \rightarrow X$ (with suitable domain); if $G=I$ is the identity operator, we write $\mathrm{e}^{t D_{F}} u_{0}=\mathscr{E}_{F}\left(t, u_{0}\right)$ and $D_{F} u_{0}=F\left(u_{0}\right)$ for short. Using that $\mathscr{E}_{F}\left(0, u_{0}\right)=u_{0}$ as well as $\frac{\mathrm{d}}{\mathrm{d} t} \mathscr{E}_{F}\left(t, u_{0}\right)=F\left(\mathscr{E}_{F}\left(t, u_{0}\right)\right)$, an application of the chain rule yields

$$
\begin{aligned}
\left.\frac{\mathrm{d}}{\mathrm{d} t}\right|_{t=0} \mathrm{e}^{t D_{F}} G u_{0} & =\left.\frac{\mathrm{d}}{\mathrm{d} t}\right|_{t=0} G\left(\mathscr{E}_{F}\left(t, u_{0}\right)\right)=\left.G^{\prime}\left(\mathscr{E}_{F}\left(t, u_{0}\right)\right) F\left(\mathscr{E}_{F}\left(t, u_{0}\right)\right)\right|_{t=0}=G^{\prime}\left(u_{0}\right) F\left(u_{0}\right) \\
& =D_{F} G u_{0} ;
\end{aligned}
$$

thus, in accordance with the identity $L=\left.\frac{\mathrm{d}}{\mathrm{d} t}\right|_{t=0} \mathrm{e}^{t L}$, which holds true for instance for any bounded linear operator $L: X \rightarrow X$ with the exponential function defined by the power series $\mathrm{e}^{t L}=\sum_{j=0}^{\infty} \frac{1}{j !} t^{j} L^{j}$, we may also set

$$
D_{F}=\left.\frac{\mathrm{d}}{\mathrm{d} t}\right|_{t=0} \mathrm{e}^{t D_{F}}
$$

Then, the defining relation for the Lie derivative is a consequence of the first relation in (A.2c). 


\section{A.2. Basic manipulation rules}

Clearly, the evolution operator forms a local one-parameter group

$$
\mathrm{e}^{(t+s) D_{F}}=\mathrm{e}^{t D_{F}} \mathrm{e}^{s D_{F}}=\mathrm{e}^{s D_{F}} \mathrm{e}^{t D_{F}}, \quad 0 \leq t+s \leq T,\left.\quad \mathrm{e}^{t D_{F}}\right|_{t=0}=I,
$$

since $\mathscr{E}_{F}\left(t+s, u_{0}\right)=\mathscr{E}_{F}\left(s, \mathscr{E}_{F}\left(t, u_{0}\right)\right)$ by the local existence and uniqueness of the solution and consequently $\mathrm{e}^{(t+s) D_{F}} G u_{0}=G\left(\mathscr{E}_{F}\left(t+s, u_{0}\right)\right)=G\left(\mathscr{E}_{F}\left(s, \mathscr{E}_{F}\left(t, u_{0}\right)\right)\right)=\mathrm{e}^{t D_{F}} \mathrm{e}^{s D_{F}} G u_{0}$. In regard to the general scheme (2.2) of an exponential operator splitting method, it should also be noted that the composition of evolution operators acts in reversed order, i.e., it holds

$$
\mathrm{e}^{t D_{F_{1}}} \mathrm{e}^{s D_{F_{2}}} G u_{0}=G\left(\mathscr{E}_{F_{2}}\left(s, \mathscr{E}_{F_{1}}\left(t, u_{0}\right)\right)\right), \quad 0 \leq t+s \leq T .
$$

Moreover, the following linearity and scaling relations are valid

$$
\begin{gathered}
D_{F_{1}+F_{2}}=D_{F_{1}}+D_{F_{2}}, \quad D_{c F}=c D_{F}, \\
D_{F}\left(G_{1}+G_{2}\right)=D_{F} G_{1}+D_{F} G_{2}, \quad D_{F}(c G)=c D_{F} G, \\
\mathrm{e}^{t D_{F}}\left(G_{1}+G_{2}\right)=\mathrm{e}^{t D_{F}} G_{1}+\mathrm{e}^{t D_{F}} G_{2}, \quad \mathrm{e}^{t D_{F}}(c G)=c \mathrm{e}^{t D_{F}} G, \quad 0 \leq t \leq T, \\
\mathrm{e}^{t D_{c F}}=\mathrm{e}^{c t D_{F}}, \quad 0 \leq t \leq T,
\end{gathered}
$$

for any complex scalar $c$. But, in general, $\mathrm{e}^{t D_{F}} G u_{0}$ and $D_{F} G u_{0}$ are nonlinear with respect to $u_{0}$.

\section{A.3. Derivatives}

Besides, we employ the relation

$$
\frac{\mathrm{d}}{\mathrm{d} t} \mathrm{e}^{t D_{F}}=D_{F} \mathrm{e}^{t D_{F}}=\mathrm{e}^{t D_{F}} D_{F}, \quad 0 \leq t \leq T,
$$

which allows to rewrite the initial value problem (A.1) as

$$
\frac{\mathrm{d}}{\mathrm{d} t} \mathrm{e}^{t D_{F}} u_{0}=D_{F} \mathrm{e}^{t D_{F}} u_{0}=\mathrm{e}^{t D_{F}} D_{F} u_{0}, \quad 0 \leq t \leq T,\left.\quad \mathrm{e}^{t D_{F}}\right|_{t=0} u_{0}=u_{0} .
$$

The above identity is verified by the following calculation

$$
\begin{aligned}
D_{F} \mathrm{e}^{t D_{F}} G u_{0} & =G^{\prime}\left(\mathscr{E}_{F}\left(t, u_{0}\right)\right) \partial_{2} \mathscr{E}_{F}\left(t, u_{0}\right) F\left(u_{0}\right)=\left.G^{\prime}\left(\mathscr{E}_{F}\left(t, u_{0}\right)\right) \frac{\mathrm{d}}{\mathrm{d} s}\right|_{s=0} \mathscr{E}_{F}\left(t, \mathscr{E}_{F}\left(s, u_{0}\right)\right) \\
& =\left.G^{\prime}\left(\mathscr{E}_{F}\left(t, u_{0}\right)\right) \frac{\mathrm{d}}{\mathrm{d} s}\right|_{s=0} \mathscr{E}_{F}\left(t+s, u_{0}\right)=G^{\prime}\left(\mathscr{E}_{F}\left(t, u_{0}\right)\right) F\left(\mathscr{E}_{F}\left(t, u_{0}\right)\right)=\mathrm{e}^{t D_{F}} D_{F} G u_{0}, \quad 0 \leq t \leq T,
\end{aligned}
$$

or, in brief, $D_{F} \mathrm{e}^{t D_{F}}=\left.\frac{\mathrm{d}}{\mathrm{d} s}\right|_{s=0} \mathrm{e}^{s D_{F}} \mathrm{e}^{t D_{F}}=\left.\frac{\mathrm{d}}{\mathrm{d} s}\right|_{s=0} \mathrm{e}^{(t+s) D_{F}}=\mathrm{e}^{t D_{F}} D_{F}$. Hereby, we denote by $\partial_{2} \mathscr{E}_{F}$ the derivative of the evolution operator with respect to the initial value; we recall that $\mathscr{E}_{F}$ and $\partial_{2} \mathscr{E}_{F}$ solve the initial value problems

$$
\begin{gathered}
\frac{\mathrm{d}}{\mathrm{d} t} \mathscr{E}_{F}\left(t, u_{0}\right)=F\left(\mathscr{E}_{F}\left(t, u_{0}\right)\right), \quad 0 \leq t \leq T,\left.\quad \mathscr{E}_{F}\left(t, u_{0}\right)\right|_{t=0}=u_{0}, \\
\frac{\mathrm{d}}{\mathrm{d} t} \partial_{2} \mathscr{E}_{F}\left(t, u_{0}\right)=F^{\prime}\left(\mathscr{E}_{F}\left(t, u_{0}\right)\right) \partial_{2} \mathscr{E}_{F}\left(t, u_{0}\right), \quad 0 \leq t \leq T,\left.\quad \partial_{2} \mathscr{E}_{F}\left(t, u_{0}\right)\right|_{t=0}=I .
\end{gathered}
$$

To justify manipulations below, we further note that the identity

$$
\mathrm{e}^{t D_{F}}=I+\left.\mathrm{e}^{\tau D_{F}}\right|_{\tau=0} ^{t}=I+\int_{0}^{t} \frac{\mathrm{d}}{\mathrm{d} \tau} \mathrm{e}^{\tau D_{F}} \mathrm{~d} \tau=I+\int_{0}^{t} \mathrm{e}^{\tau D_{F}} D_{F} \mathrm{~d} \tau, \quad 0 \leq t \leq T,
$$

which is justified by the above considerations, implies the expansion

$$
\mathrm{e}^{t D_{F}}=\sum_{j=0}^{k-1} \frac{1}{j !} t^{j} D_{F}^{j}+\int_{T_{k}} \mathrm{e}^{\tau_{k} D_{F}} D_{F}^{k} \mathrm{~d} \tau, \quad 0 \leq t \leq T, \quad k \geq 1,
$$


where we denote $T_{k}=\left\{\tau=\left(\tau_{1}, \tau_{2}, \ldots, \tau_{k}\right) \in \mathbb{R}^{k}: 0 \leq \tau_{k} \leq \ldots \leq \tau_{1} \leq \tau_{0}=t\right\}$ and, as common usage, set $D_{F}^{0}=I$. For the stepwise expansion of the splitting operator, it is useful to employ the recurrence relation

$$
\varphi_{j}\left(t D_{F}\right)=\frac{1}{j !} I+\varphi_{j+1}\left(t D_{F}\right) t D_{F}, \quad j \geq 0,
$$

with $\varphi_{0}\left(t D_{F}\right)=\mathrm{e}^{t D_{F}}$; in particular, for $j=0$ we retain $(\mathrm{A} .3 \mathrm{~g})$.

\section{A.4. Iterated Lie-commutators}

The Lie-commutator of two nonlinear operators is given by $\operatorname{ad}_{F}(G) v=[F, G](v)=F^{\prime}(v) G(v)-G^{\prime}(v) F(v)$; in particular, whenever $F$ and $G$ are linear, the above relation reduces to $\operatorname{ad}_{F}(G)=F G-G F$, since $F^{\prime}(v)=F$ as well as $G^{\prime}(v)=G$. In accordance with the above definition, we further set

$$
\operatorname{ad}_{D_{F}}\left(D_{G}\right) v=\left[D_{F}, D_{G}\right] v=D_{F} D_{G} v-D_{G} D_{F} v,
$$

whence $\operatorname{ad}_{D_{F}}\left(D_{G}\right)=-\operatorname{ad}_{F}(G)$. Moreover, higher iterated Lie-commutators are defined by induction

$$
\operatorname{ad}_{D_{F}}^{0}\left(D_{G}\right)=D_{G}, \quad \operatorname{ad}_{D_{F}}^{j}\left(D_{G}\right)=\left[D_{F}, \operatorname{ad}_{D_{F}^{j-1}}\left(D_{G}\right)\right], \quad j \geq 1 ;
$$

they naturally arise in our local error expansion, see (2.4c).

\section{A.5. Nonlinear variation-of-constants formula}

An essential tool in the derivation of the local error representation for high-order splitting methods is the nonlinear variation-of-constants formula.

Theorem A.1 (Gröbner-Alekseev formula). The solutions of the initial value problems

$$
\begin{gathered}
u^{\prime}(t)=F(u(t)), \quad 0 \leq t \leq T, \quad u(0)=u_{0}, \\
u^{\prime}(t)=F(u(t))+R(u(t)), \quad 0 \leq t \leq T, \quad u(0)=u_{0},
\end{gathered}
$$

are related through the nonlinear variation-of-constants formula

$$
\mathscr{E}_{F+R}\left(t, u_{0}\right)=\mathscr{E}_{F}\left(t, u_{0}\right)+\int_{0}^{t} \partial_{2} \mathscr{E}_{F}\left(t-\tau, \mathscr{E}_{F+R}\left(\tau, u_{0}\right)\right) R\left(\mathscr{E}_{F+R}\left(\tau, u_{0}\right)\right) \mathrm{d} \tau, \quad 0 \leq t \leq T,
$$

which in formal notation takes the form

$$
\mathrm{e}^{t D_{F+R}} u_{0}=\mathrm{e}^{t D_{F}} u_{0}+\int_{0}^{t} \mathrm{e}^{\tau D_{F+R}} D_{R} \mathrm{e}^{(t-\tau) D_{F}} u_{0} \mathrm{~d} \tau, \quad 0 \leq t \leq T .
$$

Proof. With the help of basic definitions and manipulation rules, see (A.3), we obtain

$$
\begin{aligned}
\frac{\mathrm{d}}{\mathrm{d} \tau} \mathrm{e}^{\tau D_{F+R}} \mathrm{e}^{(t-\tau) D_{F}} G u_{0}= & \frac{\mathrm{d}}{\mathrm{d} \tau} G\left(\mathscr{E}_{F}\left(t-\tau, \mathscr{E}_{F+R}\left(\tau, u_{0}\right)\right)\right) \\
= & G^{\prime}\left(\mathscr{E}_{F}\left(t-\tau, \mathscr{E}_{F+R}\left(\tau, u_{0}\right)\right)\right)\left(-F\left(\mathscr{E}_{F}\left(t-\tau, \mathscr{E}_{F+R}\left(\tau, u_{0}\right)\right)\right)\right. \\
& \left.+\partial_{2} \mathscr{E}_{F}\left(t-\tau, \mathscr{E}_{F+R}\left(\tau, u_{0}\right)\right)\left(F\left(\mathscr{E}_{F+R}\left(\tau, u_{0}\right)\right)+R\left(\mathscr{E}_{F+R}\left(\tau, u_{0}\right)\right)\right)\right) \\
= & G^{\prime}\left(\mathscr{E}_{F}\left(t-\tau, \mathscr{E}_{F+R}\left(\tau, u_{0}\right)\right)\right) \partial_{2} \mathscr{E}_{F}\left(t-\tau, \mathscr{E}_{F+R}\left(\tau, u_{0}\right)\right) R\left(\mathscr{E}_{F+R}\left(\tau, u_{0}\right)\right) \\
= & \mathrm{e}^{\tau D_{F+R}} D_{R} \mathrm{e}^{(t-\tau) D_{F}} G u_{0},
\end{aligned}
$$

which is in accordance with the formal calculation

$$
\frac{\mathrm{d}}{\mathrm{d} \tau} \mathrm{e}^{\tau D_{F+R}} \mathrm{e}^{(t-\tau) D_{F}}=\mathrm{e}^{\tau D_{F+R}}\left(D_{F+R}-D_{F}\right) \mathrm{e}^{(t-\tau) D_{F}}=\mathrm{e}^{\tau D_{F+R}} D_{R} \mathrm{e}^{(t-\tau) D_{F}} .
$$


As a consequence, using that

$$
\begin{aligned}
\mathrm{e}^{t D_{F+R}} G u_{0}-\mathrm{e}^{t D_{F}} G u_{0} & =\left.\mathrm{e}^{\tau D_{F+R}} \mathrm{e}^{(t-\tau) D_{F}} G u_{0}\right|_{\tau=0} ^{t}=\int_{0}^{t} \frac{\mathrm{d}}{\mathrm{d} \tau} \mathrm{e}^{\tau D_{F+R}} \mathrm{e}^{(t-\tau) D_{F}} G u_{0} \mathrm{~d} \tau \\
& =\int_{0}^{t} \mathrm{e}^{\tau D_{F+R}} D_{R} \mathrm{e}^{(t-\tau) D_{F}} G u_{0} \mathrm{~d} \tau, \quad 0 \leq t \leq T,
\end{aligned}
$$

the desired result follows when setting $G=I$.

To illustrate (2.4c), we determine the first partial derivative of the function

$$
\begin{aligned}
g_{3}\left(\tau_{1}, \tau_{2}, \tau_{3}\right) & =\mathrm{e}^{\tau_{3} D_{A}} D_{B} \mathrm{e}^{\left(\tau_{2}-\tau_{3}\right) D_{A}} D_{B} \mathrm{e}^{\left(\tau_{1}-\tau_{2}\right) D_{A}} D_{B} \mathrm{e}^{\left(t-\tau_{1}\right) D_{A}} v \\
& =\left.\frac{\mathrm{d}^{3}}{\mathrm{~d} \sigma_{1} \mathrm{~d} \sigma_{2} \mathrm{~d} \sigma_{3}}\right|_{\sigma_{1}=\sigma_{2}=\sigma_{3}=0} \mathrm{e}^{\tau_{3} D_{A}} \mathrm{e}^{\sigma_{3} D_{B}} \mathrm{e}^{\left(\tau_{2}-\tau_{3}\right) D_{A}} \mathrm{e}^{\sigma_{2} D_{B}} \mathrm{e}^{\left(\tau_{1}-\tau_{2}\right) D_{A}} \mathrm{e}^{\sigma_{1} D_{B}} \mathrm{e}^{\left(t-\tau_{1}\right) D_{A}} v
\end{aligned}
$$

with respect to $\tau_{2}$, see also (2.4a). For this purpose, we first consider

$$
\widetilde{g}\left(\tau_{2}\right)=\mathrm{e}^{\tau_{2} D_{A}} \mathrm{e}^{\sigma_{2} D_{B}} \mathrm{e}^{-\tau_{2} D_{A}} G v=G\left(\mathscr{E}_{A}\left(-\tau_{2}, \mathscr{E}_{B}\left(\sigma_{2}, \mathscr{E}_{A}\left(\tau_{2}, v\right)\right)\right)\right) ;
$$

for simplicity, we neglect the dependence of $\widetilde{g}$ on $\sigma_{2}$. On the one hand, a brief calculation shows that

$$
\begin{aligned}
\frac{\mathrm{d}}{\mathrm{d} \tau_{2}} G( & \left.\mathscr{E}_{A}\left(-\tau_{2}, \mathscr{E}_{B}\left(\sigma_{2}, \mathscr{E}_{A}\left(\tau_{2}, v\right)\right)\right)\right) \\
= & G^{\prime}\left(\mathscr{E}_{A}\left(-\tau_{2}, \mathscr{E}_{B}\left(\sigma_{2}, \mathscr{E}_{A}\left(\tau_{2}, v\right)\right)\right)\right)\left(-A\left(\mathscr{E}_{A}\left(-\tau_{2}, \mathscr{E}_{B}\left(\sigma_{2}, \mathscr{E}_{A}\left(\tau_{2}, v\right)\right)\right)\right)\right. \\
& \left.+\partial_{2} \mathscr{E}_{A}\left(-\tau_{2}, \mathscr{E}_{B}\left(\sigma_{2}, \mathscr{E}_{A}\left(\tau_{2}, v\right)\right)\right) \partial_{2} \mathscr{E}_{B}\left(\sigma_{2}, \mathscr{E}_{A}\left(\tau_{2}, v\right)\right) A\left(\mathscr{E}_{A}\left(\tau_{2}, v\right)\right)\right)
\end{aligned}
$$

on the other hand, with the help of the identity $\partial_{2} \mathscr{E}_{A}\left(-\tau_{2}, w\right) A(w)=A\left(\mathscr{E}_{A}\left(-\tau_{2}, w\right)\right)$, we obtain

$$
\begin{aligned}
& \mathrm{e}^{\tau_{2} D_{A}} D_{A} \mathrm{e}^{\sigma_{2} D_{B}} \mathrm{e}^{-\tau_{2} D_{A}} G v \\
&=G^{\prime}\left(\mathscr{E}_{A}\left(-\tau_{2}, \mathscr{E}_{B}\left(\sigma_{2}, \mathscr{E}_{A}\left(\tau_{2}, v\right)\right)\right)\right) \partial_{2} \mathscr{E}_{A}\left(-\tau_{2}, \mathscr{E}_{B}\left(\sigma_{2}, \mathscr{E}_{A}\left(\tau_{2}, v\right)\right)\right) \\
& \times \partial_{2} \mathscr{E}_{B}\left(\sigma_{2}, \mathscr{E}_{A}\left(\tau_{2}, v\right)\right) A\left(\mathscr{E}_{A}\left(\tau_{2}, v\right)\right) \\
& \mathrm{e}^{\tau_{2} D_{A}} \mathrm{e}^{\sigma_{2} D_{B}} D_{A} \mathrm{e}^{-\tau_{2} D_{A}} G v \\
&= G^{\prime}\left(\mathscr{E}_{A}\left(-\tau_{2}, \mathscr{E}_{B}\left(\sigma_{2}, \mathscr{E}_{A}\left(\tau_{2}, v\right)\right)\right)\right) \partial_{2} \mathscr{E}_{A}\left(-\tau_{2}, \mathscr{E}_{B}\left(\sigma_{2}, \mathscr{E}_{A}\left(\tau_{2}, v\right)\right)\right) A\left(\mathscr{E}_{B}\left(\sigma_{2}, \mathscr{E}_{A}\left(\tau_{2}, v\right)\right)\right) \\
&= G^{\prime}\left(\mathscr{E}_{A}\left(-\tau_{2}, \mathscr{E}_{B}\left(\sigma_{2}, \mathscr{E}_{A}\left(\tau_{2}, v\right)\right)\right)\right) A\left(\mathscr{E}_{A}\left(-\tau_{2}, \mathscr{E}_{B}\left(\sigma_{2}, \mathscr{E}_{A}\left(\tau_{2}, v\right)\right)\right)\right)
\end{aligned}
$$

which verifies the identity $\frac{\mathrm{d}}{\mathrm{d} \tau_{2}} \widetilde{g}\left(\tau_{2}\right)=\mathrm{e}^{\tau_{2} D_{A}}\left[D_{A}, \mathrm{e}^{\sigma_{2} D_{B}}\right] \mathrm{e}^{-\tau_{2} D_{A}} G v$ and further implies

$$
\partial_{\tau_{2}} g_{3}(\tau)=\mathrm{e}^{\tau_{3} D_{A}} D_{B} \mathrm{e}^{\left(\tau_{2}-\tau_{3}\right) D_{A}}\left[D_{A}, D_{B}\right] \mathrm{e}^{\left(\tau_{1}-\tau_{2}\right) D_{A}} D_{B} \mathrm{e}^{\left(t-\tau_{1}\right) D_{A}} v
$$

In a similar manner, the general case (2.4c) follows by induction.

\section{REFERENCES}

[1] J. Abhau and M.Thalhammer, A numerical study of adaptive space and time discretisations for Gross-Pitaevskii equations. J. Comput. Phys. 231 (2012) 6665-6681.

[2] R.A. Adams, Sobolev Spaces. Academic Press, Orlando, Fla. (1975).

[3] W. Bao, D. Jaksch and P. Markowich, Numerical solution of the Gross-Pitaevskii equation for Bose-Einstein condensation. J. Comput. Phys. 187 (2003) 318-342. 
[4] W. Bao and J. Shen, A fourth-order time-splitting Laguerre-Hermite pseudospectral method for Bose-Einstein condensates. SIAM J. Sci. Comput. 26 (2005) 2010-2028.

[5] C. Bardos, I. Catto, N. Mauser and S. Trabelsi, Global-in-time existence of solutions to the multiconfiguration time-dependent Hartree-Fock equations: A sufficient condition. Appl. Math. Lett. 22 (2009) 147-152.

[6] C. Bardos, I. Catto, N. Mauser and S. Trabelsi, Setting and analysis of the multi-configuration time-dependent Hartree-Fock equations. Arch. Ration. Mech. Anal. 198 (2010) 273-330.

[7] M.H. Beck, A. Jäckle, G.A. Worth, and H.-D. Meyer, The multiconfiguration time-dependent Hartree (MCTDH) method: A highly efficient algorithm for propagating wavepackets. Phys. Rep. 324 (2000) 1-105.

[8] M.H. Beck and H.-D. Meyer, An efficient and robust integration scheme for the equations of the multiconfiguration timedependent Hartree (MCTDH) method. Z. Phys. D 42 (1997) 113-129.

[9] S. Blanes and P.C. Moan, Practical symplectic partitioned Runge-Kutta and Runge-Kutta-Nyström methods. J. Comput. Appl. Math. 142 (2002) 313-330.

[10] S.C. Brenner and L.R. Scott, The Mathematical Theory of Finite Element Methods. Springer Verlag, New York, 2nd edition (2002).

[11] I. Burghardt, H.-D. Meyer and L.S. Cederbaum, Approaches to the approximate treatment of complex molecular systems by the multiconfiguration time-dependent Hartree method. J. Chem. Phys. 111 (1999) 2927-2939.

[12] J. Caillat, J. Zanghellini, M. Kitzler, W. Kreuzer, O. Koch and A. Scrinzi, Correlated multielectron systems in strong laser pulses - an MCTDHF approach. Phys. Rev. A 71 (2005) 012712.

[13] M. Caliari, Ch. Neuhauser and M. Thalhammer, High-order time-splitting Hermite and Fourier spectral methods for the Gross-Pitaevskii equation. J. Comput. Phys. 228 (2009) 822-832.

[14] S. Descombes and M. Thalhammer, An exact local error representation of exponential operator splitting methods for evolutionary problems and applications to linear Schrödinger equations in the semi-classical regime. BIT Numer. Math. 50 (2010) 729-749.

[15] P.A.M. Dirac, Note on exchange phenomena in the Thomas atom. Proc. Cambridge Philos. Soc. 26 (1930) $376-385$.

[16] J. Frenkel, Wave Mechanics, Advanced General Theory. Clarendon Press, Oxford (1934).

[17] L. Gauckler, Convergence of a split-step Hermite method for the Gross-Pitaevskii equation. IMA J. Numer. Anal. 49 (2011) 1194-1209.

[18] E. Hairer, C. Lubich and G. Wanner, Geometric Numerical Integration. Springer Verlag, Berlin-Heidelberg-New York (2002).

[19] E. Hairer, S.P. Nørsett and G. Wanner, Solving Ordinary Differential Equations I. Springer Verlag, Berlin-Heidelberg-New York (1987).

[20] G.H. Hardy, J.E. Littlewood and G. Polya, Inequalities. Cambridge Univ. Press, Cambridge (1934).

[21] T. Kato, Perturbation Theory for Linear Operators. Springer Verlag, Berlin-Heidelberg-New York (1966).

[22] T. Kato and H. Kono, time-dependent multiconfiguration theory for electronic dynamics of molecules in an intense laser field. Chem. Phys. Lett. 392 (2004) 533-540.

[23] M. Kitzler, J. Zanghellini, Ch. Jungreuthmayer, M. Smits, A. Scrinzi and T. Brabec, Ionization dynamics of extended multielectron systems. Phys. Rev. A $\mathbf{7 0}$ (2004) 041401(R).

[24] O. Koch, The variational splitting method for the multi-configuration time-dependent Hartree-Fock equations for atoms. To appear in J. Numer. Anal. Indust. Appl. Math. 7 (2012) 1-13.

[25] O. Koch, W. Kreuzer and A. Scrinzi, MCTDHF in ultrafast laser dynamics. AURORA TR-2003-29, Inst. Appl. Math. Numer. Anal., Vienna Univ. of Technology, Austria (2003). Available at http://www.othmar-koch.org/research.html.

[26] O. Koch, W. Kreuzer and A. Scrinzi, Approximation of the time-dependent electronic Schrödinger equation by MCTDHF. Appl. Math. Comput. 173 (2006) 960-976.

[27] O. Koch and C. Lubich, Regularity of the multi-configuration time-dependent Hartree approximation in quantum molecular dynamics. M2AN Math. Model. Numer. Anal. 41 (2007) 315-331.

[28] O. Koch and C. Lubich, Analysis and time integration of the multi-configuration time-dependent Hartree-Fock equations in electron dynamics. ASC Report 4/2008, Inst. Anal. Sci. Comput. Vienna Univ. of Technology (2008).

[29] O. Koch and C. Lubich, Variational splitting time integration of the MCTDHF equations in electron dynamics. IMA J. Numer. Anal. 31 (2011) 379-395.

[30] Y. Kwon, D.M. Ceperley and R.M. Martin, Effects of backflow correlation in the three-dimensional electron gas: Quantum Monte Carlo study. Phys. Rev. B 58 (1998) 6800-6806.

[31] L.D. Landau and E.M. Lifshitz, Quantum Mechanics: Non-Relativistic Theory. Pergamon Press, Oxford-New York, 3rd edition (1977).

[32] C. Lubich, A variational splitting integrator for quantum molecular dynamics. Appl. Numer. Math. 48 (2004) $355-368$.

[33] C. Lubich, From Quantum to Classical Molecular Dynamics: Reduced Models and Numerical Analysis. Zurich Lect. Adv. Math. Europ. Math. Soc., Zurich (2008).

[34] C. Lubich, On splitting methods for Schrödinger-Poisson and cubic nonlinear Schrödinger equations. Math. Comp. 77 (2008) $2141-2153$.

[35] R. McLachlan and R. Quispel, Splitting methods. Acta Numer. 11 (2002) 341-434.

[36] H.-D. Meyer, F. Gatti and G.A. Worth, editors. Multidimensional Quantum Dynamics: MCTDH Theory and Applications. Wiley-VCH, Weinheim, Berlin (2009).

[37] H.-D. Meyer, U. Manthe and L.S. Cederbaum, The multi-configurational time-dependent Hartree approach. Chem. Phys. Lett. 165 (1990) 73-78. 
[38] H.-D. Meyer and G.A. Worth, Quantum molecular dynamics: Propagating wavepackets and density operators using the multi-configuration time-dependent Hartree (MCTDH) method. Theo. Chem. Acc. 109 (2003) 251-267.

[39] M. Miklavčič, Applied Functional Analysis and Partial Differential Equations. World Scientific, Singapore (1998).

[40] I. Nagy, R. Diez Muiño, J.I. Juaristi and P.M. Echenique, Spin-resolved pair-distribution functions in an electron gas: A scattering approach based on consistent potentials. Phys. Rev. B 69 (2004) 233105.

[41] M. Nest and T. Klamroth, Correlated many-electron dynamics: Application to inelastic electron scattering at a metal film. Phys. Rev. A $\mathbf{7 2}$ (2005) 012710.

[42] M. Nest, T. Klamroth and P. Saalfrank, The multiconfiguration time-dependent Hartree-Fock method for quantum chemical calculations. J. Chem. Phys. 122 (2005) 124102.

[43] C. Neuhauser and M. Thalhammer, On the convergence of splitting methods for linear evolutionary Schrödinger equations involving an unbounded potential. BIT Numer. Math. 49 (2009) 199-215.

[44] V.M. Perez-Garcia and X. Liu, Numerical methods for the simulation of trapped nonlinear Schrödinger systems. Appl. Math. Comput. 144 (2003) 215-235.

[45] J.C. Slater, Quantum Theory of Molecules and Solids. McGraw-Hill, New York, Toronto, London 1 (1960).

[46] G. Strang, On the construction and comparison of difference schemes. SIAM J. Numer. Anal. 5 (1968) 506-517.

[47] C. Sulem and P.-L. Sulem, The Nonlinear Schrödinger Equation. Appl. Math. Sci. Springer Verlag, New York (1999).

[48] M. Thalhammer, High-order exponential operator splitting methods for time-dependent Schrödinger equations. SIAM J. Numer. Anal. 46 (2008) 2022-2038.

[49] M. Thalhammer, Convergence analysis of high-order time-splitting pseudo-spectral methods for nonlinear Schrödinger equations. SIAM J. Numer. Anal. 50 (2012) 3231-3258.

[50] S. Trabelsi, Solutions of the multiconfiguration time-dependent Hartree-Fock equations with Coulomb interactions. C. R. Acad. Sci. Paris, Ser. I 345 (2007) 145-150.

[51] H.F. Trotter, On the product of semi-groups of operators. Proc. Amer. Math. Soc. 10 (1959) 545-551.

[52] J. Zanghellini, M. Kitzler, T. Brabec and A. Scrinzi, Testing the multi-configuration time-dependent Hartree-Fock method. J. Phys. B: At. Mol. Phys. 37 (2004) 763-773.

[53] J. Zanghellini, M. Kitzler, C. Fabian, T. Brabec and A. Scrinzi, An MCTDHF approach to multi-electron dynamics in laser fields. Laser Phy. 13 (2003) 1064-1068. 